\title{
1 Tenuivirus uses a molecular bridge strategy to overcome 2 insect midgut barriers for virus persistent transmission
}

3 Gang $\mathrm{Lu}^{1}$, Shuo $\mathrm{Li}^{2}$, Changwei Zhou ${ }^{1}$, Xin Qian ${ }^{1}$, Qing Xiang ${ }^{1}$, Tongqing Yang ${ }^{1}$,

4 Jianxiang $\mathrm{Wu}^{3}$, Xueping Zhou ${ }^{3,4}$, Yijun Zhou ${ }^{2 *}$, Xin Shun Ding ${ }^{1}$ and Xiaorong Tao ${ }^{1 *}$

$6{ }^{1}$ Department of Plant Pathology, Nanjing Agricultural University, Nanjing 210095 ,

$7 \quad$ P.R. China

$8{ }^{2}$ Institute of Plant Protection, Jiangsu Academy of Agricultural Sciences, Jiangsu

9 Technical Service Center of Diagnosis and Detection for Plant Virus Diseases,

10 Nanjing 210014, P.R. China

$11{ }^{3}$ State Key Laboratory of Rice Biology, Institute of Biotechnology, Zhejiang

12 University, Hangzhou 310029, P.R. China

$13{ }^{4}$ State Key Laboratory for Biology of Plant Diseases and Insect Pests, Institute of

14 Plant Protection, Chinese Academy of Agricultural Sciences, Beijing 100193, P.R.

15 China

16 To whom correspondence should be addressed. Email: taoxiaorong@njau.edu.cn

17 (X.T.) or yjzhou@jass.ac.cn (Y.Z.).

18 Running title

19 A molecular bridge model for virus persistent transmission 


\section{Abstract}

21 Many persistent transmitted plant viruses, including Rice stripe tenuivirus (RSV), cause serious damages to crop productions in China and worldwide. Although many reports have indicated that successful insect-mediated virus transmission depends on proper virus-insect vector interactions, the mechanism(s) controlling interactions between viruses and insect vectors for virus persistent transmission remained poorly understood. In this study, we used RSV and its small brown planthopper (SBPH) vector as a working model to elucidate the molecular mechanism controlling RSV virion entrance into SBPH midgut for persistent transmission. We have now

29 demonstrated that this non-enveloped Tenuivirus uses its non-structural glycoprotein NSvc2 as a helper component to bridge the specific interaction between virion and

31 SBPH midgut cells, leading to overcome SBPH midgut barriers for virus persistent transmission. In the absence of this glycoprotein, purified RSV virion is not capable

33 of entering SBPH midgut cells. In RSV-infected cells, glycoprotein NSvc2 is 34 processed into two mature proteins: an amino-terminal protein $\mathrm{NSvc} 2-\mathrm{N}$ and a 35 carboxyl-terminal protein NSvc2-C. We determined that NSvc2-N interacted with RSV virion and bound directly to midgut lumen surface via its $\mathrm{N}$-glycosylation sites.

37 Upon recognition by midgut cells, the midgut cells underwent endocytosis followed by compartmentalizing RSV virion and NSvc2 into early and then late endosomes.

39 The acidic condition inside the late endosome triggered conformation change of 40 NSvc2-C and caused cell membrane fusion via its highly conserved fusion loop 41 motifs, leading to the release of RSV virion from endosome into cytosol. In summary, 
our results showed for the first time that a rice Tenuivirus uses a molecular bridge strategy to ensure proper interactions between virus and insect midgut for successful persistent transmission.

\section{Author summary}

Over $75 \%$ of the known plant viruses are insect transmitted. Understanding how plant viruses interacted with their insect vectors during virus transmission is one of the key steps to manage virus diseases worldwide. Both the direct and indirect virus-insect vector interaction models have been proposed for virus non-persistent and semi-persistent transmission. However, the indirect virus-vector interaction mechanism during virus persistent transmission has not been reported previously. In this study, we developed a new reverse genetics technology and demonstrated that the circulative and propagative transmitted Rice stripe tenuivirus utilizes a glycoprotein NSvc2 as a helper component to ensure a specific interaction between Tenuivirus virion and midgut cells of small brown planthopper (SBPH), leading to conquering the midgut barrier of SBPH. This is the first report of a helper component mediated-molecular bridge mechanism for virus persistent transmission. These new findings and our new model on persistent transmission expand our understanding of molecular mechanism(s) controlling virus-insect vector interactions during virus transmission in nature. 


\section{Introduction}

65 Arthropod insects play critical roles in epidemics of numerous animal and plant

66 viruses [1-3]. Based on the mode of transmission, plant viruses can be classified into

67 non-persistent, semi-persistent or persistent transmitted viruses [4-6]. For

68 non-persistent and semi-persistent transmissions, plant viruses retain only inside

69 insect stylets or on foregut surface for a short period of time. Upon probing or feeding

70 on a new host plant, viruses are quickly injected into plant cells, together with insect

71 saliva [7-9]. The persistent transmitted plant viruses (non-propagative or propagative)

72 are required to enter insect vector bodies, and then circulate and/or replicate inside the

73 vectors for several days to weeks. These persistent transmitted viruses need to pass

74 insect midgut barrier, dissemination barrier, and then salivary gland barrier prior to be

75 transmitted to new host plants $[6,10,11]$. Midgut is often considered to be one of

76 major barriers for successful persistent transmissions of plant viruses. During the

77 process of passing through barriers inside vectors, proper interactions between viruses

78 and vectors are needed for successful transmission. However, the mechanism

79 controlling the interactions between persistent transmitted plant viruses and their

80 insect vector midgut barriers remains poorly understood.

81 Molecular bridge mechanism has been reported for non-persistent and

82 semi-persistent transmitted plant viruses, respectively [4, 12, 13]. For example, virion

83 of non-persistent or semi-persistent transmitted plant viruses were reported to interact

84 with the cuticular proteins in the mouthparts or the foreguts [14, 15], and these 

to be non-transmissible by vector insects [36-38]. RSV also encodes a glycoprotein

virus-insect interactions required virally encoded non-structural helper factors as molecular bridges $[12,13]$. Viruses in the genus Potyvirus are known to encode a helper component proteinase (HC-Pro) and this HC-Pro protein acts as a molecular bridge for potyvirus virion-aphid vector interactions [16-18]. Members in the genus Caulimovirus encode a different helper factor that helps virion to retain on insect maxillary stylet [19-21]. Although virion of multiple persistent transmitted plant viruses [e.g., Luteovirus [22, 23], Geminivirus [24, 25], Reovirus [26, 27], Tospovirus [28, 29], and plant Rhabdovirus [30, 31]] were also reported to bind directly to insect midgut cells, these bindings all depended on virion surface-exposed proteins. To date, no persistent transmitted (non-propagative or propagative) plants viruses has been reported for the requirement of additional helper proteins for the transmission.

Rice stripe tenuivirus (RSV) is known to be transmitted by small brown planthopper $(\mathrm{SBPH})$ in a circulative and propagative manner, and causes severe rice losses in China and many other countries in Asia [32, 33]. The genome sequence of plant infecting Tenuivirus is similar to members of animal infecting Phlebovirus in the order of Bunyavirales. Most of the members in the order of Bunyavirales produce membrane-enveloped spherical virion with two surface-exposed glycoproteins. These surface-exposed glycoproteins are key determinants for entering host cells or for vector transmission $[29,34,35]$. However, virion of tenuiviruses are filamentous and do not have enveloped membranes. Purified tenuivirus virion was previously reported 
107 NSvc2-N and a carboxyl-terminal part protein known as NSvc2-C [39, 40]. The RSV

108 encoded glycoprotein NSvc2 was not found in the purified virion samples [41, 42].

109 Based on the above published reports we hypothesized that Rice stripe tenuivirus

110 must use a quite different mechanism to overcome the midgut barriers for its insect

111 transmission.

112 To validate this hypothesis, we conducted various experiments using RSV and

113 SBPH as our working model. We have now determined for the first time that the rice

114 Tenuivirus uses a unique molecular bridge strategy to overcome insect midgut barrier

115 for virus persistent transmission. We have shown that in the absence of RSV

116 non-structural glycoprotein NSvc2, RSV virion was unable to enter SBPH midgut

117 cells. We found that this RSV non-structural glycoprotein NSvc2 acts as a critical

118 molecular bridge to mediate the interaction between RSV virion and SBPH midgut.

119 NSvc2-N, a processed product from NSvc2, interacts with RSV virion and binds

120 directly to the midgut barrier. Upon the successful interaction, midgut cells undergo

121 endocytosis followed by compartmentalization of RSV virion and NSvc2 complexes

122 in early and then late endosomes. NSvc2-C, another processed product from NSvc2,

123 triggers membrane fusion under the acidic condition inside the late endosomes to

124 release RSV virion into cytosol. These new findings expand our understanding of

125 interactions between virion and insect vectors during transmissions of plant and

126 animal viruses.

\section{$127 \quad$ Results}




\section{Association of NSve2 protein with RSV virion in midgut of SBPH}

129 To examine whether RSV NSvc2 plays role(s) in circulative RSV transmission, we

130 first conducted a time course study on the co-localization of NSvc2 and RSV virion in

131 the midgut of SBPH during acquisition. SBPHs were fed on RSV-infected rice

132 seedlings and then collected at 4, 8, 16 and $24 \mathrm{~h}$ after feeding (30 SBPHs at each time

133 point), respectively. The collected insects were dissected and analyzed for the

134 presence of NSvc2 and RSV virion by double-immunolabeling methods using an

135 antibody against the amino-terminal NSvc2 (NSvc2-N) or RSV virion-surface

136 nucleocapsid protein (NP). As shown in Fig $1 \mathrm{~A}$ that, after $4 \mathrm{~h}$ feeding on the

137 RSV-infected rice seedlings, numerous RSV virion (green) had accumulated in the

138 midgut lumen. In the same tissues, NSvc2 was also detected (red) and found to

139 co-localize with RSV virion on the actin-labelled intestinal microvillus (blue) (Fig

140 1A). The overlapped coefficient (OC) value for the red and green labeling signal was

$1410.76 \pm 0.03$ at $4 \mathrm{~h}$ post feeding (Fig 1E), indicating that NSvc2 and RSV virion were

142 localized close to each other. At $8 \mathrm{~h}$ post feeding, NSvc2 was found to co-localize

143 with RSV virion in various sized vesicles-like structures in epithelial cells (Fig 1B).

144 Analysis of OC value showed again that NSvc2 and RSV were indeed localized close

145 to each other (Fig 1F). At 16 h post feeding, RSV virion was detected together with

146 NSvc2 in cytoplasm of midgut epithelial cells, with an OC value of $0.73 \pm 0.06$ (Fig

$1471 \mathrm{C}$ and $1 \mathrm{G})$. Even at $24 \mathrm{~h}$ post feeding, NSvc2 was still associated with RSV virion,

148 with an OC value of $0.76 \pm 0.04$, and the virus had spread into the surrounding midgut

149 epithelial cells (Fig 1D and 1H). These data showed that RSV-encoded glycoprotein 
NSvc2 was associated with RSV virion in SBPH midgut during insect feeding on

151 RSV-infected rice plants.

NSvc2 protein is critical for RSV virion entrance into SBPH midgut

153 RSV virion was purified from virus-infected rice seedlings through ultracentrifugation

154 using a $20 \%$ glycerol cushion. After ultracentrifugation, four different fractions

155 starting from the top of the upper supernatant phase (Sup1 to Sup4), four fractions

156 starting from the top of the lower $20 \%$ glycerol phase (Gly1 to Gly4), and the pellet

157 (Pel) were collected and analyzed individually by immunoblotting assays using an

158 antibody against RSV NP or NSvc2-N (Fig 2A and 2B). Results showed that the pellet

159 sample contained RSV virion, and the four supernatant fractions (Sup1 to Sup4)

160 contained NSvc2 protein. In contrast, the four glycerol fractions (Gly1 to Gly4)

161 contained RSV virion and NSvc2 protein (Fig 2B). Transmission Electron Microscopy

162 showed that numerous filamentous RSV virion were present in the pellet sample (Fig

$1632 \mathrm{C})$.

164 To verify the above findings, SBPHs were fed on a mixture of sucrose and the

165 combined supernatant fraction, combined glycerol fraction, the resuspended pellet

166 sample, or the mixed supernatant and pellet sample through a layer of stretched

167 parafilm membrane for $24 \mathrm{~h}$. As shown in Fig 2D and 2E, NSvc2 (red) and RSV

168 virion (green) were detected together in the epithelial cells of SBPHs fed on the

169 mixture of sucrose and the combined glycerol fraction (Fig 2D, row 2). RSV virion

170 was, however, not detected in the microvillus of SBPHs fed on the mixtures of 
171 sucrose and the combined supernatant fraction or the resuspended pellet sample (Fig

172 2D, row 1 and 3). In contrast, when insects were allowed to feed on a mixture of

173 sucrose and the combined supernatant fraction plus the resuspended pellet sample,

174 both NSvc2 and RSV virion were detected in the epithelial cells (OC value $=0.95 \pm$

175 0.03; Fig 2D, row 4), similar to that found for the SBPHs fed on the mixture of

176 sucrose and the combined glycerol fraction $(\mathrm{OC}$ value $=0.97 \pm 0.04)$. Statistical

177 analysis of RSV transmission using SBPHs fed on various samples also indicated that

178 feeding on the mixture of sucrose and the combined supernatant fraction plus the

179 resuspended pellet sample allowed RSV virion entrance into the midgut epithelial

180 cells for a successful virus transmission (Fig 2E). This finding suggests that RSV

181 NSvc2 is a critical factor mediating RSV virion entrance into SBPH midgut cells. To

182 validate the interaction between RSV NP and NSvc2, we performed yeast two-hybrid

183 assays. Results showed that RSV NP interacted with both NSvc2-N and NSvc2-C (Fig

184 2F). All these data suggested that NSvc2 protein is critical for RSV virion entrance

185 into SBPH midgut.

186 Recombinant amino-terminal soluble region of NSvc2 directly binds

187 to midgut and inhibits subsequent RSV acquisition by SBPH

188 Previous studies have shown that NSvc2 can be further processed into two mature

189 glycoproteins, namely amino-terminal and carboxyl-terminal NSvc2 [40]. S1A Fig

190 illustrated the predicted structure of NSvc2 and the positions of its signal peptides,

191 transmembrane regions, and the predicted glycan sites. To examine the potential roles 
192 of amino-terminal NSvc2 (NSvc2-N) in RSV transmission, we expressed the soluble

193 NSvc2-N protein (referenced to as NSvc2-N:S) in Sf9 insect cells using a

194 recombinant baculovirus expression system (S1B Fig). After purification using the

195 Ni-NTA agarose, the expression of the recombinant NSvc2-N:S was confirmed by

196 Western blot assay using an anti-NSvc2-N polyclonal antibody (Fig 3A).

197 To further determine whether the recombinant NSvc2-N:S protein can bind midgut

198 epidermal microvillus, SBPHs were allowed to feed on purified NSvc2-N:S for $3 \mathrm{~h}$

199 followed by a $12 \mathrm{~h}$ feeding on a sucrose solution to remove unbound NSvc2-N:S.

200 Results of immunofluorescence analyses showed that NSvc2-N:S (green signal) could

201 be readily detected in the midgut lumen near the surface of epithelial cells in the

202 alimentary canal (Fig 3B). As a negative control, SBPHs were allowed to feed on

203 Tomato spotted wilt virus (TSWV) encoded glycoprotein (Gn:S), known to bind thrip

204 midgets [28]. As expected, the TSWV Gn:S (green) was not detected in SBPH 205 midguts.

206 Based on the above results, we further hypothesized that the pre-acquired

207 NSvc2-N:S could prevent RSV acquisition by blocking midgut RSV specific

208 receptors. To test this hypothesis, SBPHs were allowed to feed on the purified

209 NSvc2-N:S for $24 \mathrm{~h}$ and then on RSV-infected rice plants for $48 \mathrm{~h}$. The alimentary

210 canals were dissected from SBPHs and probed using the RSV NP or NSvc2-N

211 specific antibodies. Under the confocal microscope, the labeled RSV virion was found

212 in the midgut lumen of SBPHs pre-fed with purified NSvc2-N:S (S2A Fig),

213 suggesting that RSV virion was prevented from entering into the midgut epithelial 
214 cells. In contrast, RSV virion was detected in the midgut epithelial cells after the

215 insects were pre-fed with TSWV Gn:S or with sucrose alone (S2B Fig and S2C Fig).

216 To further confirm the role of NSvc2-N during SBPH acquisition of RSV, SBPHs

217 pre-fed with NSvc2-N:S were allowed to feed on RSV-infected rice plants for $48 \mathrm{~h}$

218 and then on healthy rice seedlings for 12 days. After this feeding period, the insects

219 were tested for RSV infection by ELISA assay. Results showed that pre-feeding

220 SBPHs with NSvc2-N:S did significantly reduce the rate of RSV infection compared

221 with the insects pre-fed with TSWV Gn:S or sucrose only (Fig 3C). This finding

222 indicated that NSvc2-N:S could inhibit RSV entrance into SBPH midgut.

$223 \mathrm{~N}$-glycosylation of $\mathbf{N S v c 2}-\mathrm{N}$ is required for midgut receptor 224 recognition of $\mathrm{RSV}$

225 Computer-assisted modeling suggested that NSvc2 might be modified through

226 glycosylation (S1A Fig). To confirm this prediction, purified NSvc2-N:S was

227 incubated with PNGaseF (a N-glycosidase) or O-Glycosidases and Neuraminidase

228 (O-Gly + Neur) to remove the $\mathrm{N}$ - or O-linked glycans, respectively. Subsequent

229 SDS-PAGE and immunoblotting analyses showed that the purified NSvc2-N:S protein

230 band was shifted in the gel after the PNGaseF treatment, compared with the

231 non-treated NSvc2-N:S (Fig 3D, compare lane1 and 2). No clear band shift was

232 detected when NSvc2-N:S was treated with O-Gly + Neur (Fig 3D, compare lane1

233 and 3). When NSvc2-N:S was treated with PNGaseF together with O-Gly + Neur, the

234 protein band shifted as that treated with PNGaseF (Fig 3D, compare lane2 and 4), 
235 indicating that NSvc2-N was modified by the N-linked glycans.

236 We then generated a NSvc2-N:S site-directed alanine-substitution mutant,

237 NSvc2-N:S ${ }^{\text {N114A/N199A/N232A }}(\mathrm{N} 114 \mathrm{~A} / \mathrm{N} 199 \mathrm{~A} / \mathrm{N} 232 \mathrm{~A})$, at its putative N-linked glycan

238 sites, and a mutant, NSvc2-N:S $\mathrm{S}^{\mathrm{S} 8 \mathrm{~A} / \mathrm{S} 128 \mathrm{~A} / \mathrm{S} 183 \mathrm{~A}}(\mathrm{~S} 38 \mathrm{~A} / \mathrm{S} 128 \mathrm{~A} / \mathrm{S} 183 \mathrm{~A})$, at its putative

239 O-linked glycan sites. These two mutants were purified as describe above for the

240 wild-type (WT) NSvc2-N:S followed by the enzymatic deglycosylation analyses.

241 Results showed that, without PNGaseF treatment, the NSvc2-N:S ${ }^{\text {N114A/N199A/N232A }}$

242 mutant displayed a similar protein band shift in the gel as that shown by the WT

243 NSvc2-N:S treated with PNGaseF (Fig 3E, compare lane 2 and 3). A slight band shift

244 was noticed for the NSvc2-N:S $\mathrm{S}^{\mathrm{N} 114 \mathrm{~A} / \mathrm{N} 199 \mathrm{~A} / \mathrm{N} 232 \mathrm{~A}}$ mutant, without or with PNGaseF

245 treatment (Fig 3E, compare lane 3 and 4). No O-linked glycan modification was

246 detected for the NSvc2-N:S ${ }^{\mathrm{S} 38 \mathrm{~A} / \mathrm{S} 128 \mathrm{~A} / \mathrm{S} 183 \mathrm{~A}}$ mutant (S38A/S128A/S183A, Fig 3F). This

247 result indicated that residue N114, N199 and N232 of NSvc2-N:S are indeed the

$248 \quad \mathrm{~N}$-glycosylation sites.

249 To investigate whether the N-linked glycosylation can affect the recognition of

250 NSvc2-N:S by midgut surface receptors, SBPHs were fed with the two mutant

251 proteins, respectively, for $3 \mathrm{~h}$ followed by a $12 \mathrm{~h}$ feeding on a sucrose only solution to

252 clean insect alimentary canals. Insects fed with the NSvc2-N:S ${ }^{\mathrm{N} 114 \mathrm{~A} / \mathrm{N} 199 \mathrm{~A} / \mathrm{N} 232 \mathrm{~A}}$ mutant

253 protein showed almost no green labeling signal at the surface of midgut microvillus.

254 In contrast, insects fed with the NSvc2-N:S $\mathrm{S}^{\mathrm{S} 38 \mathrm{~A} / \mathrm{S} 128 \mathrm{~A} / \mathrm{S} 183 \mathrm{~A}}$ mutant protein did (Fig $3 \mathrm{G}$ ).

255 ELISA results showed that the NSvc2-N:S $\mathrm{S}^{\mathrm{N} 114 \mathrm{~A} / \mathrm{N} 199 \mathrm{~A} / \mathrm{N} 232 \mathrm{~A}}$ mutant protein had no

256 obvious effect on SBPH RSV acquisition (Fig 3H). Consequently, we conclude that 

surface receptor recognition.

261 from endosomes

262 Endocytosis is an important process for several circulative-transmitted animal viruses

263 during entering into animal cells [43]. The localization of NSvc2-N and RSV virion

264 suggested that RSV virion and NSvc2-N has entered into endosomal-like vesicle (Fig

$2651 B$ and $1 \mathrm{~F})$. To further investigate the localization of RSV virion and NSvc2-N after

266 recognition by SBPH midgut cell receptor(s), early and late endosome specific

267 markers (Rab5, EEA1 and Rab7) were used to visualize these vesicles [44, 45].

268 Results showed that RSV virion did co-localize with the early endosome Rab5 marker

269 and the late endosome Rab7 marker (Fig 4A and 4B). In the same study, NSvc2-N and

270 carboxyl-terminal protein of NSvc2 (NSvc2-C) were also found to co-localize with 271 early endosome EEA1 marker (Fig 4C and 4D).

272 To examine the potential role(s) of NSvc2-C, SBPHs were allowed to feed on

273 RSV-infected rice plants for 4, 8, 16 or $24 \mathrm{~h}$, and then used for immunofluorescence

274 labeling assays. NSvc2-C with red labeling signal was observed together with RSV

275 virion (green) at the surface of microvillus (blue) (S3A Fig and S3E Fig; OC value = $2760.86 \pm 0.04$ ), and in the endosomal-like vesicles in epithelial cells (S3B Fig and S3F 277 Fig; OC value $=0.95 \pm 0.03$ ) at 4 and $8 \mathrm{~h}$ post feeding. At 16 and $24 \mathrm{~h}$ post feeding, 
278 RSV virion (green) had accumulated alone in the cytoplasm of epithelial cells (S3C

279 Fig and S3G Fig [OC value $=0.18 \pm 0.02]$, and S3D Fig and S3H Fig [OC value $=$

$2800.32 \pm 0.06])$, suggesting that NSvc2-C remained inside the endosomal-like vesicles

281 while RSV virion was released from the endosomal-like vesicles and accumulated in

282 the cytoplasm. To confirm this finding, the localization of RSV virion, NSvc2-N and

283 NSvc2-C was examined just at the time that RSV virion release from endosomes. The

284 results showed that after being released from endosomes, NSvc2-N continued to

285 associate with RSV virion in cytosol (Fig 4E, white dashed circles), whereas NSvc2-C

286 stayed inside the actin-labeled endosome and was not released into cytosol of

287 epithelial cells (Fig 4F, white dashed box). This finding indicates that NSvc2-C

288 associates with RSV virion:NSvc2-N in endosome but was separated from them after

289 the virion were released from endosome.

NSvc2-C induces insect cell membrane fusion under acidic conditions

291 To determine whether NSvc2-N and/or NSvc2-C play roles in membrane fusion, we

292 fused a signal peptide of baculovirus (gp64) to NSvc2-N and NSvc2-C, and expressed

293 these proteins individually in insect Spodoptera frugiperda (Sf9) cells by the

294 recombinant baculovirus expression system (Fig 5A). Expressions of these

295 recombinant proteins were confirmed by an immunolabeling assay. Under the laser

296 scanning confocal microscope, red labeling fluorescence signal representing NSvc2-N

297 or NSvc2-C was observed on the Sf9 cell membranes (Fig 5B). We then tested

298 whether NSvc2-N or NSvc2-C could trigger cell membrane fusion under acidic 
conditions. Sf9 cells infected with the recombinant NSvc2-N or NSvc2-C baculovirus were treated with a PBS, $\mathrm{pH} 5.0$, for 2 min and then grown in a $\mathrm{pH}$ neutral medium.

301 Numerous cell-cell fusions (syncytium) were observed at $4 \mathrm{~h}$ post acidic PBS 302 treatment of Sf9 cells infected with the NSvc2-C baculovirus (Fig 5E and 5G). In 303 contrast, no significant cell-cell fusion was observed for cells infected with the 304 NSvc2-N or empty baculovirus (Fig 5C and 5D). In addition, cell membrane fusion 305 was observed in cells co-infected with NSvc2-N and NSvc2-C baculoviruses (Fig 5F 306 and 5G), confirming that RSV NSvc2-C, but not NSvc2-N, played an important role 307 in cell-cell fusion under acidic conditions.

308 Conserved hydrophobic fusion-loop motifs in NSvc2-C are crucial for 309 fusogenic activity

310 To further investigate the function of NSvc2-C in cell membrane fusion, we generated

311 a three-dimensional (3D) structure of NSvc2-C through a homology modeling 312 approach (S4A Fig and S4B Fig). This 3D structure consisted of three distinct 313 domains: domain I (yellow), II (red), and III (blue). Two putative fusion loops (green) 314 were found at the top of the 3D structure. Similar fusion loops were reported to be 315 responsible for cell-cell fusion during animal virus infections [46, 47]. In this study, 316 we constructed three NSvc2-C fusion domain deletion mutants (i.e., $\Delta \mathrm{FL} 1, \Delta \mathrm{FL} 2$ and $317 \Delta \mathrm{FL} 1+\Delta \mathrm{FL} 2)$, and expressed them individually in Sf9 cells followed by 318 immunoblotting (S4C Fig and S4D Fig). The fusogenic activities of these deletion 319 mutants were then examined in Sf9 cells using the recombinant baculovirus 
$\Delta \mathrm{FL} 1$ or $\triangle \mathrm{FL} 2$ mutant was much less than that induced by the WT NSvc2-C (S4E Fig roles in cell-cell fusion.

To identify the amino acid residue(s) important for fusogenic activity, we aligned the RSV fusion loop sequences (Cys459-Cys465, Loop1 and Cys485-Tyr498, Loop 2)

328 with the loop sequences of other four tenuiviruses (Fig 5H). The alignment result

329 indicated that these two fusion loops were relatively conserved among the five

330 tenuiviruses. Six conserved hydrophobic amino acid residues (Phe460, Phe489,

331 Phe492, Tyr494, Pro496 and Tyr498) were found at the unique vertex in the modeled

332 NSvc2-C 3D structure (Fig 5I). Introduction of mutations into these six amino acid 333 residues suggested that three residues (F460A, F489A, and Y498A) were important 334 for membrane fusion activity (Fig $5 \mathrm{~J}$ and $5 \mathrm{~K}$ ).

\section{NSvc2-C fusogenic-activity-deficient mutant fails to mediate RSV 336 virion release from endosome}

337 The above results showed that RSV NSvc2-N and NSvc2-C had different functions 338 during RSV transmission via SBPH. To elucidate the role of NSvc2-C in RSV 339 entrance into midgut cells during virus acquisition, we analyzed the fusion loop 340 mutants for their functions in RSV virion release from endosome. Purified RSV virion 
341 was incubated for $3 \mathrm{~h}$ with Sf9 cell crude extracts containing the full length NSvc2

342 and then used the mixture to feed SBPHs for $24 \mathrm{~h}$. Midguts were isolated from SBPHs

343 and probed for the presence of RSV virion and NSvc2-N through

344 immunofluorescence labeling. Result showed that RSV virion (green) and NSvc2-N

345 (red) were both present in the endosomal-like vesicles in epithelial cells, indicating

346 that both RSV virion and NSvc2-N had entered into the midgut cells (Fig 6A, upper

347 row). Result also showed that RSV virion was released from endosome at $24 \mathrm{~h}$ post

348 feeding. We then prepared Sf9 cell crude extracts containing the

$349 \mathrm{~N}$-glycosylation-deficient mutant $\mathrm{NSvc} 2^{\mathrm{N} 114 \mathrm{~A} / \mathrm{N} 199 \mathrm{~A} / \mathrm{N} 232 \mathrm{~A}}$ or the

350 fusogenic-activity-deficient mutant $\mathrm{NSvc} 2^{\mathrm{F} 460 \mathrm{~A} / \mathrm{F} 489 \mathrm{~A} / \mathrm{Y} 498 \mathrm{~A}}$. Using the same feeding

351 method described above, RSV virion was detected in the midgut lumen but not in the

352 epithelial cells of SBPHs fed with the mixture of RSV virion and

353 NSvc2 ${ }^{\text {N114A/N199A/N232A }}$ mutant (Fig 6A, middle row). This study also showed that RSV

354 virion and $\mathrm{NSvc} 2^{\mathrm{F} 460 \mathrm{~A} / \mathrm{F} 489 \mathrm{~A} / \mathrm{Y} 498 \mathrm{~A}}$ mutant overcame the midgut barrier and entered

355 together into the endosomal-like structures. However, RSV virion and

$356 \mathrm{NSvc} 2^{\mathrm{F} 460 \mathrm{~A} / \mathrm{F} 489 \mathrm{~A} / \mathrm{Y} 498 \mathrm{~A}}$ mutant were hardly detected in the cytoplasm of epithelial cells.

357 Also, greater number of endosomal-like structures was found in epithelial cells of

358 SBPHs fed with the mixture of RSV virion and NSvc2 $2^{\mathrm{F} 460 \mathrm{~A} / \mathrm{F} 489 \mathrm{~A} / \mathrm{Y} 498 \mathrm{~A}}$ mutant than that

359 fed with the mixture of RSV virion and wild type NSvc2 (Fig 6A, bottom row).

360 Statistical analysis further confirmed that RSV virion did enter endosomes but failed

361 to be released into cytosol after SBPHs were fed with the mixtures of purified RSV

362 virion and $\mathrm{NSvc} 2^{\mathrm{F} 460 \mathrm{~A} / \mathrm{F} 489 \mathrm{~A} / \mathrm{Y} 498 \mathrm{~A}}$ mutant, while RSV virion did not enter endosomes 
363 when SBPHs were fed with the mixtures of purified RSV virion and

$364 \mathrm{NSvc} 2^{\mathrm{N} 114 \mathrm{~A} / \mathrm{N} 199 \mathrm{~A} / \mathrm{N} 232 \mathrm{~A}}$ mutant $($ Fig 6B). This finding indicates that the

365 fusogenic-activity-deficient $\mathrm{NSvc} 2$ mutant can mediate virion passage through

366 epithelial cells but failed to release RSV virion from endosomal-like vesicles.

\section{Discussion}

368 Understanding how plant viruses are transmitted through their insect vectors in field is

369 one of the key steps to manage virus diseases worldwide. Insect midgut is a major

370 barrier to block the entrance of non-compatible plant viruses. In this study, we used

371 RSV and its SBPH vector as a working model to elucidate the molecular mechanism

372 controlling RSV virion entrance into SBPH midgut for replication. We demonstrated

373 here for the first time that RSV, a circulative and propagative transmitted Tenuivirus,

374 used a molecular bridge strategy to overcome the midgut barrier in SBPH for virus

375 persistent transmission.

376 In this study, we confirmed that glycoprotein NSvc2 of RSV is not a structural

377 protein of RSV virion, and found that in the absence of this glycoprotein, RSV virion

378 is unable to overcome the midgut barrier (Fig 2). RSV glycoprotein NSvc2 was

379 further processed into an amino-terminal part protein known as NSvc2-N and a

380 carboxyl-terminal part protein known as NSvc2-C in RSV-infected cells [39, 40]. We

381 found that NSvc2-N accumulated on midgut surface during virus acquisition by SBPH.

382 We also found that the ectopically expressed and purified soluble NSvc2-N:S bound

383 directly to midgut surface and inhibited the subsequent RSV acquisition by SBPH. We 
consider that this soluble NSvc2-N:S protein can recognize SBPH midgut surface receptor(s). Our enzymatic deglycosylation result showed that NSvc2-N could be modified by N-linked but not O-linked glycans. The glycan-modification of NSvc2-N might be different from the N- and O-glycosylation of TSWV Gn protein reported 388 previously [28]. It is noteworthy that the $\mathrm{N}$-glycosylation-deficient $389 \mathrm{NSvc2}^{\mathrm{N} 114 \mathrm{~A} / \mathrm{N} 199 \mathrm{~A} / \mathrm{N} 232 \mathrm{~A}}$ mutant was unable to interact with midgut surface and to 390 block RSV acquisition by SBPH. Moreover, feeding SBPHs with a mixture of 391 purified RSV virion and crude extract from Sf9 cells expressing full length NSvc2 resulted in a successful transmission of RSV. In contrast, the

393 N-glycosylation-deficient NSvc2 mutant was unable to facilitate RSV virion to pass

394 through SBPH midgut barrier. Therefore, we propose that $\mathrm{N}$-glycosylation 395 modification plays an important role in interaction between RSV virion and SBPH 396 midgut receptor(s). A sugar transporter in SBPH midgut cell was recently found to 397 play critical role in RSV transmission [48]. It would be interested in investigating the 398 interactions between NSvc2 and sugar transporter protein and the biological function 399 of glycosylation for these interactions in the future.

400 Although the function of RSV glycoprotein NSvc2 was proposed to be similar to 401 that of tospovirus glycoprotein or phytoreovirus spike protein during virus acquisition 402 and transmission [29, 49], NSvc2 is not present on the surface of non-enveloped 403 filamentous RSV virion while both tospovirus glycoprotein and phytoreovirus spike 404 protein are located virion surface. Purified RSV virion is not capable of entering 405 SBPH midgut cells. This inability can, however, be rescued by addition of 
406 glycoprotein NSvc2 (Fig 2D and Fig 6A). For non-persistent or semi-persistent

407 transmitted plant viruses, the helper component proteins or helper factors were shown

408 to act as molecular bridges for the interactions between virion and the cuticle of their

409 insect vectors [4, 6]. Although these helper component proteins or factors are not

410 located on the surface of purified virion, these factors are absolutely required for

411 insect transmissions $[18,50]$. Our results presented in this paper indicate that this

412 helper factor theory can also be used to explain the function of glycoprotein NSvc2

413 during RSV circulative and propagative transmission by SBPH. First, the RSV

414 glycoprotein NSvc2 is not a virion structural protein (Fig 2). Second, the glycoprotein

415 NSvc2 acts as a molecular bridge to ensure the interaction between RSV virion and

416 SBPH midgut receptor(s) (Fig 3). Third, the glycoprotein NSvc2 is absolutely

417 required for RSV virion entrance into SBPH midgut cells (Fig 2 and Fig 6). These

418 findings strongly supported that RSV glycoprotein NSvc2 is a helper factor protein

419 and this helper factor functions as a molecular bridge to allow the proper interaction

420 between RSV viroin and SBPH midgut during persistent transmission of Tenuivirus.

421 After recognition and interaction with RSV virion, midgut epithelial cells

422 underwent endocytosis, resulting in an enclosure of RSV virion:NSvc2 complexes in

423 early and then late endosomes. After that, RSV virion was released from the late

424 endosomes into cytoplasm for replication and for further spreading to other cells.

425 NSvc2-C was initially found together with RSV virion on midgut surface, and later in

426 endosomal-like vesicles. Although NSvc2-C retained inside the endosomes,

427 NSvc2-N-associated RSV virion was released into cytoplasm. We determined that, 
under acidic conditions, the expressed NSvc2-C could cause Sf9 cell membrane to fuse. To identify the amino acid residue(s) controlling this membrane fusion activity, a

430 homology based modeling approach was used to create a 3D structure of NSvc2-C

431 followed by site-directed mutagenesis. With the aid of homology modeling, we 432 identified that NSvc2-C residue F460, F489 and Y498 played critical roles during cell

433 membrane fusion. Importantly, the NSvc2 mutant that failed to cause cell membrane

434 fusion was unable to release RSV virion from endosomes into cytoplasm. It is likely

435 that the acidic condition inside endosomes caused conformation change of NSvc2-C,

436 leading to cell membrane fusion. The two fusion loops found in NSvc2 are highly 437 conserved among the members in the genus Tenuivirus and are critical for cell 438 membrane fusion induction.

439 Current gene function studies for plant multi-segmented negative-strand RNA 440 viruses are difficult due mainly to the lack of proper reverse genetics methods. In this 441 paper, we described a new and useful approach to overcome this obstacle. We first 442 expressed the WT or mutant NSvc2 individually in Sf9 insect cells and then incubated 443 the Sf9 cell crude extracts with purified RSV virion. After feeding SBPHs with a WT 444 or mutant NSvc2 extract mixed with purified RSV virion, we successfully determined 445 that virion from the sample containing the WT NSvc2 and purified RSV virion 446 overcame the midgut barrier, entered the epithelial cells, and then released from late 447 endosomes to cytosol for further replication and transmission. The defective NSvc2 448 mutants failed to interact with midgut surface and thus were unable to mediate the 449 entrance of RSV virion into epithelial cells. In addition, using this approach, we were 
450 able to confirm that the cell membrane fusion defective mutant could not mediate the

451 release of RSV virion from endosomes to cytosol. The assay methods developed in

452 this study should benefit gene function studies for viruses whose infectious clones are

453 currently difficult to make. We proposed that this newly developed technology can not

454 only be used to investigate the functions of tenuivirus glycoproteins, but can also be

455 used to determine the functions of glycoproteins encoded by other plant

456 multi-segmented negative-strand RNA viruses.

457 Taken together, we conclude that circulative and propagative transmitted RSV uses

458 a molecular bridge strategy to bring RSV virion to SBPH midgut surface during

459 vector transmission. Based on the findings presented in this study, we propose a

460 working model for plant tenuiviruses (Fig 6C). In this model, the NSvc2-N and

461 NSvc2-C do not associate with RSV virion in infected plant cells. After plant sap

462 containing RSV virion, NSvc2-N, and NSvc2-C is acquired into the midgut lumen of

463 SBPH, NSvc2-N recognizes the unidentified midgut cell surface receptor(s) and acts

464 as a molecular bridge to ensure the interaction between RSV virion and midgut

465 surface. Upon attachment of RSV virion:NSvc2-N:NSvc2-C complexes to midgut

466 surface receptor(s), midgut cells undergo endocytosis, resulting in

467 compartmentalization of RSV virion, NSvc2-N, and NSvc2-C in early and then late

468 endosomes. The acidic condition inside the late endosomes triggers a conformation

469 change of NSvc2-C, and the conformation-changed NSvc2-C cause cell membrane to

470 fuse. Finally, the RSV virion:NSvc2-N complexes are released into cytosol (Fig 6C).

471 Findings presented in this paper demonstrate a new type of virus-vector midgut 
472 interaction that requires a virally encoded molecular bridge during virus persistent

473 transmission. This type of mechanism has never been shown for persistent transmitted

474 plant viruses or animal viruses. This new finding expands our understanding of

475 molecular mechanism(s) controlling virus-insect vector interactions during virus

476 transmission in nature.

\section{Materials and methods}

\section{$478 \quad$ Insect and virus maintenance}

479 Rice stripe tenuivirus (RSV) was previously isolated from an RSV-infected rice plant, 480 and maintained inside a growth chamber at the Jiangsu Academy of Agricultural 481 Sciences, Jiangsu Province, China. Small brown planthopper (SBPH) was reared on 482 seedlings of rice cv. Wuyujing NO.3 inside growth incubators set at $26.5{ }^{\circ} \mathrm{C}$ and a 483 photoperiod of $16 \mathrm{~h} / 8 \mathrm{~h}$ (light / dark). Rice seedlings were changed once every 12 484 days to ensure sufficient nutrition as described [51].

485 Isolation of RSV virion

486 Eight hundred milliliter precooled 0.1 M phosphate buffer (PB), pH7.5, with $0.01 \mathrm{M}$ 487 EDTA was added to $50 \mathrm{~g}$ RSV-infected rice leaf tissues followed by $5 \mathrm{~min}$ 488 homogenization in a blender. The homogenate was centrifuged at $8,000 \times g$ for 20 $489 \min$ at $4^{\circ} \mathrm{C}$. The resulting supernatant was slowly mixed with $6 \%$ PEG 6000 and 0.1 $490 \mathrm{M} \mathrm{NaCL}$, and stirred overnight at $4{ }^{\circ} \mathrm{C}$. After centrifugation at $8,000 \times g$ for $20 \mathrm{~min}$, 491 the pellet was resuspended in $0.01 \mathrm{M} \mathrm{PB}$ and then centrifuged again at $150,000 \times g$ 
492 for $2 \mathrm{~h}$. The pellet was resuspended in $6 \mathrm{ml} 0.01 \mathrm{M} \mathrm{PB}$ buffer and laid on a $4 \mathrm{ml} 20 \%$

493 glycerol cushion inside a centrifuge tube followed by a centrifugation at $150,000 \times g$

494 for $2 \mathrm{~h}$. Different fractions inside the centrifuge tube were collected individually and 495 the pellet was resuspended in PB buffer (contains $30 \%$ glycerol) prior to storage at $496-70{ }^{\circ} \mathrm{C}$.

\section{Immunofluorescence staining of SBPH midgut organs}

498 Immunofluorescence staining assay was performed as described previously with 499 specific modifications [52]. Midguts were obtained from second-instar nymphs and 500 fixed overnight in a $4 \%$ paraformaldehyde (PFA, Thermo Fisher, USA) solution at

$5014{ }^{\circ} \mathrm{C}$. After three rinses in a $0.01 \mathrm{M}$ phosphate-buffered saline (PBS), pH 7.4, the 502 midguts were treated for $30 \mathrm{~min}$ in a $2 \%$ Triton X-100 solution followed by $1 \mathrm{~h}$ 503 incubation in a specific primary antibody. The midguts were then incubated in a 504 specific fluorescence conjugated secondary antibody or a fluorescence conjugated 505 actin antibody for $2 \mathrm{~h}$ at room temperature (RT). The midguts were rinsed three times 506 in PBS and mounted in an antifade solution (Solarbio). The mounted midguts were 507 examined under an inverted Leica TCS SP8 fluorescent confocal microscope (Leica 508 Microsystems, Solms, Germany). LAS X software was used to analyze fluorescence 509 spectra to determine co-localization of two proteins.

510 Rabbit polyclonal antibody against RSV NSvc2-N or NSvc2-C was produced in our 511 laboratory. Mouse monoclonal antibody against RSV NP was a gift from Professor 512 Jianxiang Wu, Zhejiang University, Hangzhou, China. Primary antibodies also used in 
513 this study included Rab5 anti-rabbit IgG (C8B1; Cell Signaling Technology), Rab7

514 anti-rabbit IgG (D95F2; Cell Signaling Technology), and EEA1 anti-mouse IgG

515 (NBP2-36568; Novus). Secondary antibodies used in this study were FITC

516 conjugated rabbit anti-mouse IgG (F9137; Sigma) or goat anti-rabbit IgG (F9887;

517 Sigma), TRITC conjugated goat anti-rabbit IgG (T6778; Sigma), Alexa Fluor 647

518 phalloidin (A22287; Invitrogen) or Rhodamine Phalloidin (R415; Invitrogen).

519 Recombinant baculovirus expression in insect cell

520 Construction of recombinant baculovirus was the same as described previously [53].

521 Sequence encoding RSV NSvc2-N:S (amino acid positions 20 to 265, lacking the

522 signal peptide and the transmembrane domain) was PCR-amplified using a cDNA

523 from a RSV-infected rice plant. The resulting PCR fragment represented the RSV

524 NSvc2-N:S sequence and a six-His tag sequence at its 3' end terminus. The Gp64

525 signal peptide sequence was then fused to the 5' end terminus of NSvc2-N:S sequence

526 via overlapping PCR. The final PCR product was cloned into vector pFastBac1 (S1

527 Table). The site-directed mutagenized mutants were constructed as described [54]. All

528 plasmids were sequenced, transformed individually into DH10Bac ${ }^{\mathrm{TM}}$ cells to generate

529 recombinant baculoviruses as instructed by the manufacturer (Invitrogen). The

530 recombinant baculoviruses were co-transfected individually with the FnGENE HD

531 Transfection Reagent (Promega) into Spodoptera frugiperda (Sf9) cells to obtain the

532 stable expression of recombinant baculoviruses. 
534 Sf9 cells were infected with recombinant baculoviruses at a multiplicity of infection

535 (MOI) of five, and the infected-cells were collected at $72 \mathrm{~h}$ post infection. The

536 infected-cells were lysed in $10 \mathrm{ml}$ PBS using a ultrasonic cell crusher followed by

537 centrifugation at $4{ }^{\circ} \mathrm{C}$ to remove cell debris. After centrifugation, the supernatant was

538 collected, incubated with nickel-nitrilotriacetic acid resin (Ni-NTA, Germany) for $2 \mathrm{~h}$,

539 and then loaded onto a chromatographic column (Bio-Rad Hercules, California). After

540 separation, the column was washed with two bed volumes of $50 \mathrm{mM}$ imidazole in

541 PBS, and the recombinant protein was eluted with $250 \mathrm{mM}$ imidazole followed by

542 dialysis against PBS prior to storage at $-70{ }^{\circ} \mathrm{C}$ until use.

543 Glycosylation and Immunoblotting assays

544 Purified NSvc2-N:S and various mutant proteins were deglycosylated with PNGaseF

545 as instructed (NEB, USA) to remove N-linked glycans or Neuraminidase and

546 O-Glycosidase (NEB, USA) to remove O-linked glycans. Proteins were mixed with

$5470.5 \%$ SDS and $40 \mathrm{mM} \mathrm{DTT}$, and incubated at $100^{\circ} \mathrm{C}$ for $10 \mathrm{~min}$. After denaturation,

548 buffer and glycosidases were added to the samples and incubated for $3 \mathrm{~h}$ at $37{ }^{\circ} \mathrm{C}$. The

549 enzyme-treated samples were mixed with a loading buffer containing SDS and boiled

550 for 10 min prior to electrophoresis in $10 \%$ (w/v) SDS-PAGE gels. The separated

551 proteins were transferred to nitrocellulose membranes and the membranes were

552 probed with a polyclonal rabbit antibody against NSvc2-N (1:5,000 dilution) and then

553 a goat anti-rabbit IgG HRP conjugate (31466; Invitrogen). The detection signal was

554 visualized using the ChemiDoc ${ }^{\mathrm{TM}}$ Touch Imaging System (Bio-Rad, Hercules, 
555 California).

\section{Glycoprotein midgut binding assays}

557 Midgut binding assays were performed as described previously [28]. Briefly,

558 second-instar nymphs were placed in open-ended EP centrifuge tubes and fed with 559 mixtures of purified glycoproteins resuspended in a TF buffer (PBS with $10 \%$ 560 glycerol, $0.01 \%$ Chicago sky blue, and $5 \mathrm{mg} / \mathrm{ml} \mathrm{BSA}$ ) through a stretched parafilm 561 membrane. After $3 \mathrm{~h}$ feeding, the glycoprotein mixtures were replaced with a $10 \%$ 562 sucrose solution for another $12 \mathrm{~h}$ feeding to clear midguts, indicated by the 563 disappearance of the Chicago sky blue dye from the midguts. The insects were then 564 dissected, fixed in PFA and analyzed by immunofluorescence staining as described 565 above.

\section{Detection of SBPH RSV acquisition using Enzyme linked}

\section{7 immunosorbent assay (ELISA)}

568 Second-instar nymphs were placed in an empty bottle for $2 \mathrm{~h}$ and then allowed to

569 acquire purified protein through a stretched parafilm membrane for $24 \mathrm{~h}$. The pre-fed

570 SBPHs were then allowed to feed on RSV-infected rice seedlings for $48 \mathrm{~h}$ prior to

571 feeding on healthy rice seedlings for another 12 days. SBPHs were transferred

572 individually into a centrifuge tube and grinded thoroughly in PBS buffer. After brief 573 centrifugation, individual supernatant samples were blotted on nitrocellulose 574 membranes and the membranes were probed with a 1:5,000 (v/v) diluted monoclonal 
575 antibody against RSV NP, and then a 1:20,000 diluted secondary alkaline phosphatase

576 (AP)-coupled goat anti-mouse IgG (Sigma). Detection signal was visualized using a

577 5-bromo-4-chloro-3-indo-lylphosphate-nitroblue tetrazolium (BCIP-NBT) solution

578 (Sangon Biotech, Shanghai, China). Three independent experiments with 50 nymphs /

579 treatment were performed.

\section{Yeast two-hybrid assay}

581 Yeast two-hybrid assays were performed according to the instructions from the

582 manufacturer (Clontech, TaKaRa, Japan). Briefly, one bait vector and a specific prey

583 vector, described in the result section, were co-transformed into the Y2HGold strain

584 cells by heat shock method. In addition, vector pGADT7-T was co-transformed into

585 the Y2HGold strain cells with vector pGBKT7-53 or vector pGBKT7-Lam, and used

586 as a positive and a negative control, respectively. All co-transformed cells were first

587 grown on a synthetic dextrose medium lacking Tryptophan and Leucine amino acid

588 (SD-Trp-Leu) for 3 days at $30{ }^{\circ} \mathrm{C}$, and then on a synthetic dextrose medium lacking

589 Tryptophan, Leucine, Histidine and Ademethionine amino acid (SD-Trp-Leu-His-Ade)

590 for 5 days at $30^{\circ} \mathrm{C}$.

\section{Low pH-induced membrane fusion assay}

592 Sf9 cells were infected with different recombinant baculoviruses at a MOI of five for

$59348 \mathrm{~h}$. The infected cells were washed twice with fresh medium, treated with a PBS

594 buffer, $\mathrm{pH}$ 5.0, for $2 \mathrm{~min}$ and then in a $\mathrm{pH}$ neutral medium. The cells were incubated 
bioRxiv preprint doi: https://doi org/101101/404657; this version posted August 30, 2018. The copyright holder for this preprint (which was

not certified by peer review) is the author/funder, who has granted bioRxiv a license to display the preprint in perpetuity. It is made available under aCC-BY 4.0 International license.

595 for $4 \mathrm{~h}$ at $28{ }^{\circ} \mathrm{C}$ and then examined for the cell-cell membrane fusion under an

Olympus IX71 inverted fluorescence microscope (Olympus, Hamburg, Germany).

597 Homology modeling of NSve2-C

598 Homology based modeling of NSvc2-C was as described previously [55, 56]. Briefly,

599 RSV NSvc2-C sequence was used to search the I-TASSER Server software. Based on

600 the high TM-score value (0.805), glycoprotein of Sever Fever with Thrombocytopenia

601 Syndrome Phlebovirus (SFTSV) (PDB: 5G47) was chosen as the template to build the

602 homology based model of RSV NSvc2-C. Amino acid residues and their surface

603 displays in the three-dimensional structure were predicted using the PyMOL program.

604 Acknowledgments

605 We thank Prof. Yidong Wu (College of Plant Protection, Nanjing Agricultural

606 University, China) for kindly providing the insect Sf9 cell lines. We also thank Prof.

607 Tong Zhou (Institute of Plant Protection, Jiangsu Academy of Agricultural Sciences)

608 for kindly providing the RSV-infected rice samples.

609

610 References

611 1. Gray SM, Banerjee N. Mechanisms of arthropod transmission of plant and animal viruses.

612 Microbiol Mol Biol R. 1999; 63:128-48. PMID: 10066833.

613 2. Ruckert C, Weger-Lucarelli J, Garcia-Luna SM, Young MC, Byas AD, Murrieta RA, et al. Impact 614 of simultaneous exposure to arboviruses on infection and transmission by Aedes aegypti mosquitoes. 615 Nat commun. 2017; 8: 15412. doi: 10.1038/ncomms15412 PMID: 28524874. 
3. Dader B, Then C, Berthelot E, Ducousso M, Ng JCK, Drucker M. Insect transmission of plant viruses: Multilayered interactions optimize viral propagation. Insect Sci. 2017; 24: 929-46. doi: 10.1111/1744-7917.12470 PMID: 28426155.

4. Ng JC, Falk BW. Virus-vector interactions mediating nonpersistent and semipersistent transmission of plant viruses. Annu Rev Phytopathol. 2006; 44: 183-212. doi: 10.1146/annurev.phyto.44.070505.143325 PMID: 16602948.

5. Hogenhout SA, Ammar ED, Whitfield AE, Redinbaugh MG. Insect vector interactions with persistently transmitted viruses. Annu Rev Phytopathol. 2008; 46: 327-59. doi: 10.1146/annurev.phyto.022508.092135 PMID: 18680428.

6. Whitfield AE, Falk BW, Rotenberg D. Insect vector-mediated transmission of plant viruses. Virology. 2015; 479: 278-89. doi: 10.1016/j.virol.2015.03.026 PMID: 25824478.

7. Childress SA, Harris KF. Localization of Virus-Like Particles in the Foreguts of Viruliferous Graminella-Nigrifrons Leafhoppers Carrying the Semi-Persistent Maize Chlorotic Dwarf Virus. J Gen Virol. 1989; 70: 247-51. doi: 10.1099/0022-1317-70-1-247.

8. Martin B, Collar JL, Tjallingii WF, Fereres A. Intracellular ingestion and salivation by aphids may cause the acquisition and inoculation of non-persistently transmitted plant viruses. J Gen Virol. 1997; 78: 2701-5. doi: 10.1099/0022-1317-78-10-2701 PMID: 9349493.

9. Chen AY, Walker GP, Carter D, Ng JC. A virus capsid component mediates virion retention and transmission by its insect vector. Proc Natl Acad Sci USA. 2011; 108: 16777-82. doi: 10.1073/pnas.1109384108 PMID: 21930903.

10. Blanc S, Drucker M, Uzest M. Localizing viruses in their insect vectors. Annu Rev Phytopathol. 2014; 52: 403-25. doi: 10.1146/annurev-phyto-102313-045920 PMID: 24996011.

11. Gray S, Cilia M, Ghanim M. Circulative, "nonpropagative" virus transmission: an orchestra of virus-, insect-, and plant-derived instruments. Adv Virus Res. 2014; 89: 141-99. doi: 10.1016/B978-0-12-800172-1.00004-5 PMID: 24751196.

12. Pirone TP, Blanc S. Helper-dependent vector transmission of plant viruses. Annu Rev Phytopathol. 1996; 34: 227-47. doi: 10.1146/annurev.phyto.34.1.227 PMID: 15012542.

13. Ng JCK, Zhou JS. Insect vector-plant virus interactions associated with non-circulative, semi-persistent transmission: current perspectives and future challenges. Curr Opin Virol. 2015; 15 : 48-55. doi: 10.1016/j.coviro.2015.07.006 PMID: 26318639.

14. Uzest M, Gargani D, Drucker M, Hebrard E, Garzo E, Candresse T, et al. A protein key to plant virus transmission at the tip of the insect vector stylet. Proc Natl Acad Sci USA. 2007; 104 : 17959-64. doi: 10.1073/pnas.0706608104 PMID: 17962414.

15. Webster CG, Pichon E, van Munster M, Monsion B, Deshoux M, Gargani D, et al. Identification of Plant Virus Receptor Candidates in the Stylets of Their Aphid Vectors. J Virol. 2018; 92: e00432-18. doi: 10.1128/JVI.00432-18 PMID: 29769332.

16. Atreya CD, Pirone TP. Mutational Analysis of the Helper Component-Proteinase Gene of a Potyvirus - Effects of Amino-Acid Substitutions, Deletions, and Gene Replacement on Virulence and Aphid Transmissibility. Proc Natl Acad Sci USA. 1993; 90: 11919-23. doi: 10.1073/pnas.90.24.11919 PMID: 8265648.

17. Blanc S, Ammar ED, Garcia-Lampasona S, Dolja VV, Llave C, Baker J, et al. Mutations in the potyvirus helper component protein: effects on interactions with virions and aphid stylets. J Gen Virol. 1998; 79: 3119-22. doi: 10.1099/0022-1317-79-12-3119 PMID: 9880030.

18. Ruiz-Ferrer V, Boskovic J, Alfonso C, Rivas G, Llorca O, Lopez-Abella D, et al. Structural analysis 
of tobacco etch potyvirus HC-Pro oligomers involved in aphid transmission. J Virol. 2005; 79: 3758-65. doi: 10.1128/Jvi.79.6.3758-3765.2005 PMID: 15731269.

19. Lung MC, Pirone TP. Acquisition factor required for aphid transmission of purified cauliflower mosaic virus. Virology. 1974; 60: 260-4. PMID: 4841178.

20. Leh V, Jacquot E, Geldreich A, Hermann T, Leclerc D, Cerutti M, et al. Aphid transmission of cauliflower mosaic virus requires the viral PIII protein. EMBO J. 1999; 18: 7077-85. doi: 10.1093/emboj/18.24.7077 PMID: 10601029.

21. Drucker M, Froissart R, Hebrard E, Uzest M, Ravallec M, Esperandieu P, et al. Intracellular distribution of viral gene products regulates a complex mechanism of cauliflower mosaic virus acquisition by its aphid vector. Proc Natl Acad Sci USA. 2002; 99: 2422-7. doi: 10.1073/pnas.042587799 PMID: 11842201.

22. Brault V, Vandenheuvel JFJM, Verbeek M, Zieglergraff V, Reutenauer A, Herrbach E, et al. Aphid Transmission of Beet Western Yellows Luteovirus Requires the Minor Capsid Read-through Protein P74. EMBO J. 1995; 14: 650-9. PMID: 7882968.

23. Gray S, Gildow FE. Luteovirus-aphid interactions. Annu Rev Phytopathol. 2003; 41: 539-66. doi: 10.1146/annurev.phyto.41.012203.105815 PMID: 12730400.

24. Azzam O, Frazer J, Delarosa D, Beaver JS, Ahlquist P, Maxwell DP. Whitefly transmission and efficient ssDNA accumulation of bean golden mosaic geminivirus require functional coat protein. Virology. 1994; 204: 289-96. doi: 10.1006/viro.1994.1533 PMID: 8091659.

25. Noris E, Vaira AM, Caciagli P, Masenga V, Gronenborn B, Accotto GP. Amino acids in the capsid protein of tomato yellow leaf curl virus that are crucial for systemic infection, particle formation, and insect transmission. J Virol. 1998; 72: 10050-7. PMID: 9811744.

26. Tomaru M, Maruyama W, Kikuchi A, Yan J, Zhu Y, Suzuki N, et al. The loss of outer capsid protein $\mathrm{P} 2$ results in nontransmissibility by the insect vector of rice dwarf phytoreovirus. J Virol. 1997; 71: 8019-23. PMID: 9311898.

27. Wei TY, Li Y. Rice Reoviruses in Insect Vectors. Annu Rev Phytopathol. 2016; 54: 99-120. doi: 10.1146/annurev-phyto-080615-095900 PMID: 27296147.

28. Whitfield AE, Ullman DE, German TL. Expression and characterization of a soluble form of Tomato spotted wilt virus glycoprotein $\mathrm{G}(\mathrm{N})$. J Virol. 2004; 78: 13197-206. doi: 10.1128/Jvi.78.23.13197-13206.2004 PMID: 15542672.

29. Sin SH, McNulty BC, Kennedy GG, Moyer JW. Viral genetic determinants for thrips transmission of Tomato spotted wilt virus. Proc Natl Acad Sci USA. 2005; 102: 5168-73. doi: 10.1073/pnas.0407354102 PMID: 15753307.

30. Redinbaugh MG, Hogenhout SA. Plant rhabdoviruses. Curr Top Microbiol Immunol. 2005; 292 : 143-63. PMID: 15981471

31. Ammar ED, Tsai CW, Whitfield AE, Redinbaugh MG, Hogenhout SA. Cellular and Molecular Aspects of Rhabdovirus Interactions with Insect and Plant Hosts. Annu Rev Entomol. 2009; 54: 447-68. doi: 10.1146/annurev.ento.54.110807.090454 PMID: 18793103.

32. Hibino H. Biology and epidemiology of rice viruses. Annu Rev Phytopathol. 1996; 34: 249-74. doi: 10.1146/annurev.phyto.34.1.249 PMID: 15012543.

33. Cho WK, Lian S, Kim SM, Park SH, Kim KH. Current Insights into Research on Rice stripe virus. Plant Pathol J. 2013; 29: 223-33. doi: 10.5423/PPJ.RW.10.2012.0158 PMID: 25288949.

34. Cifuentes-Munoz N, Salazar-Quiroz N, Tischler ND. Hantavirus Gn and Gc Envelope Glycoproteins: Key Structural Units for Virus Cell Entry and Virus Assembly. Viruses. 2014; 6: 
1801-22. doi: 10.3390/v6041801 PMID: 24755564.

35. Spiegel M, Plegge T, Pohlmann S. The Role of Phlebovirus Glycoproteins in Viral Entry, Assembly and Release. Viruses. 2016; 8: 202-21. doi: 10.3390/v8070202 PMID: 27455305.

36. Ramirez BC, Haenni AL. Molecular biology of tenuiviruses, a remarkable group of plant viruses. J Gen Virol. 1994; 75: 467-75. doi: 10.1099/0022-1317-75-3-467 PMID: 8126445.

37. Falk BW, Tsai JH. Biology and molecular biology of viruses in the genus Tenuivirus. Annu Rev Phytopathol. 1998; 36: 139-63. doi: 10.1146/annurev.phyto.36.1.139 PMID: 15012496.

38. Huo Y, Chen LY, Su L, Wu Y, Chen XY, Fang RX, et al. Artificial feeding Rice stripe virus enables efficient virus infection of Laodelphax striatellus. J Virol Methods. 2016; 235: 139-43. doi: 10.1016/j.jviromet.2016.06.003 PMID: 27283882.

39. Zhao S, Zhang G, Dai X, Hou Y, Li M, Liang J, et al. Processing and intracellular localization of rice stripe virus $P c 2$ protein in insect cells. Virology. 2012; 429: 148-54. doi: 10.1016/j.virol.2012.04.018 PMID: 22575054.

40. Yao M, Liu XF, Li S, Xu Y, Zhou YJ, Zhou XP, et al. Rice stripe tenuivirus NSvc2 glycoproteins targeted to the golgi body by the $\mathrm{N}$-terminal transmembrane domain and adjacent cytosolic 24 amino acids via the COP I- and COP II-dependent secretion pathway. J Virol. 2014; 88: 3223-34. doi: 10.1128/Jvi.03006-13 PMID: 24390331.

41. Toriyama S. Characterization of Rice Stripe Virus: a heavy component carrying infectivity. J Gen Virol. 1982; 61: 187-95. doi: 10.1099/0022-1317-61-2-187.

42. Toriyama S. An RNA-dependent RNA-polymerase associated with the filamentous nucleoproteins of rice stripe virus. J Gen Virol. 1986; 67: 1247-55. doi: 10.1099/0022-1317-67-7-1247.

43. Mercer J, Schelhaas M, Helenius A. Virus entry by endocytosis. Annu Rev Biochem. 2010; 79: 803-33. doi: 10.1146/annurev-biochem-060208-104626 PMID: 20196649.

44. Saeed MF, Kolokoltsov AA, Albrecht T, Davey RA. Cellular entry of ebola virus involves uptake by a macropinocytosis-like mechanism and subsequent trafficking through early and late endosomes. PLoS Pathog. 2010; 6: e1001110. doi: 10.1371/journal.ppat.1001110 PMID: 20862315.

45. Xia WQ, Liang Y, Chi Y, Pan LL, Zhao J, Liu SS, et al. Intracellular trafficking of begomoviruses in the midgut cells of their insect vector. PLoS Pathog. 2018; 14: e1006866. doi: 10.1371/journal.ppat.1006866 PMID: 29370296.

46. Podbilewicz B. Virus and cell fusion mechanisms. Annu Rev Cell Dev Biol. 2014; 30: 111-39. doi: 10.1146/annurev-cellbio-101512-122422 PMID: 25000995.

47. Halldorsson S, Behrens AJ, Harlos K, Huiskonen JT, Elliott RM, Crispin M, et al. Structure of a phleboviral envelope glycoprotein reveals a consolidated model of membrane fusion. Proc Natl Acad Sci USA. 2016; 113: 7154-9. doi: 10.1073/pnas.1603827113 PMID: 27325770.

48. Qin FL, Liu WW, Wu N, Zhang L, Zhang ZK, Zhou XP, et al. Invasion of midgut epithelial cells by a persistently transmitted virus is mediated by sugar transporter 6 in its insect vector. PLoS Pathog. 2018; 14: e1007201. doi: 10.1371/journal.ppat.1007201 PMID: 30052679.

49. Zhou F, Pu Y, Wei T, Liu H, Deng W, Wei C, et al. The P2 capsid protein of the nonenveloped rice dwarf phytoreovirus induces membrane fusion in insect host cells. Proc Natl Acad Sci USA. 2007; 104: 19547-52. doi: 10.1073/pnas.0708946104 PMID: 18042708.

50. Hoh F, Uzest M, Drucker M, Plisson-Chastang C, Bron P, Blanc S, et al. Structural insights into the molecular mechanisms of cauliflower mosaic virus transmission by its insect vector. J Virol. 2010; 84: 4706-13. doi: 10.1128/jvi.02662-09 PMID: 20181714.

51. Li S, Wang S, Wang X, Li X, Zi J, Ge S, et al. Rice stripe virus affects the viability of its vector 
offspring by changing developmental gene expression in embryos. Sci Rep. 2015; 5:7883. doi: 10.1038/srep07883 PMID: 25601039.

750 52. Chen Q, Chen HY, Mao QZ, Liu QF, Shimizu T, Uehara-Ichiki T, et al. Tubular structure induced by a plant virus facilitates viral spread in its vector insect. PLoS Pathog. 2012; 8: e1003032. doi: 10.1371/journal.ppat.1003032 PMID: 23166500.

53. Kost TA, Condreay JP, Jarvis DL. Baculovirus as versatile vectors for protein expression in insect and mammalian cells. Nat Biotechnol. 2005; 23: 567-75. doi: 10.1038/nbt1095 PMID: 15877075.

54. Zhu M, Jiang L, Bai BH, Zhao WY, Chen XJ, Li J, et al. The intracellular immune receptor sw-5b confers broad-spectrum resistance to tospoviruses through recognition of a conserved 21-amino acid viral effector epitope. Plant Cell. 2017; 29: 2214-32. doi: 10.1105/tpc.17.00180 PMID: 75828814646.

55. Li J, Feng Z, Wu J, Huang Y, Lu G, Zhu M, et al. Structure and function analysis of nucleocapsid protein of tomato spotted wilt virus interacting with RNA using homology modeling. J Biol Chem. 2015; 290: 3950-61. doi: 10.1074/jbc.M114.604678 PMID: 25540203.

56. Lu G, Li J, Zhou YJ, Zhou XP, Tao XR. Model-based structural and functional characterization of the Rice stripe tenuivirus nucleocapsid protein interacting with viral genomic RNA. Virology. 2017; 506: 73-83. doi: 10.1016/j.virol.2017.03.010 PMID: 28359901. 
766

767

768

769

770

771

772

773

774

775

776

777

778

779

780

781

782

783

784

785

\section{Figure Legends}

Fig 1. NSvc2 associates with RSV virion in SBPH midgut. (A) NSvc2 (Red) and

RSV virion (green) were co-localized in midgut lumen and on the surface of intestinal microvillus (blue) at $4 \mathrm{~h}$ post feeding on RSV-infected rice plants. The presence of RSV virion, NSvc2, and actin were detected with antibodies specific for RSV NP, NSvc2-N, or actin. The boxed regions are shown on the right side of the corresponding images with three panels. The detection signal for NSvc2 is in red, the detection single for virion is in green and the merged detection signal is in yellow. (B) Co-localization of NSvc2 and RSV virion in vesicle-like structures in epithelial cells at $8 \mathrm{~h}$ post feeding. (C) Co-localization of NSvc2 and RSV virion in cytoplasm of epithelial cells at $16 \mathrm{~h}$ post feeding. (D) NSvc2 and RSV virion complexes were detected in epithelial cells of midgut at $24 \mathrm{~h}$ post feeding. (E-H) Analyses of overlapped fluorescence spectra from NSvc2 (red) and RSV virion (green) at different stages. Fluorescence signals were from the white dashed line indicated areas. The overlap coefficient (OC) values were determined individually using the LAS X software. ML, midgut lumen; EC, epithelial cell; Bar, $25 \mu \mathrm{m}$.

\section{Fig 2. NSve2 protein is required for RSV entrance into SBPH midgut. (A) NSvc2}

is absent in purified RSV virion. Extract from RSV-infected rice plants was loaded on the top of $4 \mathrm{~mL} 20 \%$ glycerol. The supernatant fractions, glycerol fractions and the pellet were collected after ultra-centrifugation. (B) The fractions were analyzed in gels followed by Coomassie blue staining or by immunoblotting using antibodies specific for RSV NP or NSvc2-N. Sizes of the protein bands are shown on the left. Asterisk 
789 indicates a RSV NP dimer. (C) Morphology of RSV virion in a resuspended pellet

790 sample examined by Electron Microscopy. Arrows indicate filamentous RSV virion.

791 Bar, $50 \mathrm{~nm}$. (D) Immunofluorescence labeling of NSvc2 (red) and RSV virion (green)

792 in SBPH midguts after feeding with the combined supernatant fraction, glycerol

793 fraction, resuspended pellet or the supernatant fraction and resuspended pellet mixture.

794 The boxed regions are enlarged and shown on the right side of the merged images.

795 Overlapping fluorescence spectra analyses were done for the white dashed line

796 indicated areas shown in the right panels. The overlap coefficient (OC) values were

797 determined using the LAS X software. ML, midgut lumen; EC, epithelial cell; Bar, 25

$798 \mu \mathrm{m}$. (E) Statistic analysis of RSV infection in SBPH after feeding on different

799 fractions. Each bar represents three independent biological repeats from each

800 experiment ( $\mathrm{n}=50 /$ group). ${ }^{* *}, p<0.01$ by student $t$-test analysis. (F) Yeast

801 two-hybrid assay for the interactions between RSV NP and NSvc2-N, or between

802 RSV NP and NSvc2-C. RSV NP was fused to a GAL4 activation domain (AD-NP),

803 and NSvc2-N or NSvc2-C was fused to a GAL4 binding domain (BD-NSvc2-N,

804 BD-NSvc2-C). Yeast cells were co-transformed with indicated plasmids, and were

805 assayed for protein interactions on synthetic dextrose -Trp/-Leu/-His/-Ade medium.

806

807 Fig 3. N-glycosylation of NSvc2-N is essential for the recognition of midgut

808 surface receptor. (A) Immunoblot analysis of NSvc2-N:S expression in Sf9 cells.

809 Sizes of protein markers are indicated on the left and the NSvc2-N:S protein band is

810 indicated with an arrow. (B) Binding of purified NSvc2-N:S to microvillus surface of 
811 SBPH midgut. Arrows indicate the accumulation of NSvc2-N:S (green, left panel) in

812 midgut lumen. Purified TSWV Gn:S was used as a negative control in this study

813 (right panel). ML, midgut lumen; EC, epithelial cell; Bar, $25 \mu \mathrm{m}$. (C) Percentages of

814 RSV acquisition by SBPHs pre-fed with NSvc2-N:S, TSWV Gn:S, or sucrose only.

815 All experiments were performed three times (50 SBPHs for each group). $* * p<0.01$

816 by $t$-test analysis. (D-F) Enzymatic de-glycosylation of NSvc2-N:S. Purified

817 NSvc2-N:S was incubated with PNGaseF or O-Glycosidase + Neuraminidase (O-Gly

$818+$ Neur) to determine the types of glycans (D). PNGaseF was used to remove the

819 N-linked glycans, and O-Gly + Neur were used to remove the O-linked glycans.

820 N-glycosylation (E) and O-glycosylation (F) of the triple asparagine mutant

821 (NSvc2-N:S $\mathrm{S}^{\mathrm{N} 114 \mathrm{~A} / \mathrm{N} 199 \mathrm{~A} / \mathrm{N} 232 \mathrm{~A}}$ ) or triple serine mutant (NSvc2-N:S $\mathrm{S}^{\mathrm{S} 38 \mathrm{~A} / \mathrm{S} 128 \mathrm{~A} / \mathrm{S} 183 \mathrm{~A}}$ ) were

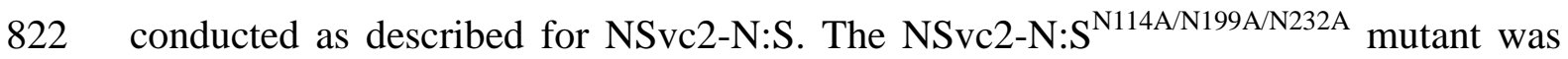

823 treated with $\mathrm{PNGaseF}$ while the NSvc2-N:S ${ }^{\mathrm{S} 38 \mathrm{~A} / \mathrm{S} 128 \mathrm{~A} / \mathrm{S} 183 \mathrm{~A}}$ mutant was treated with

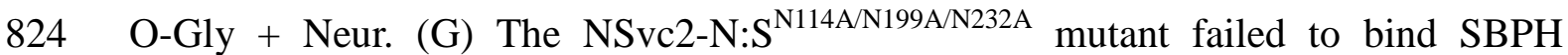

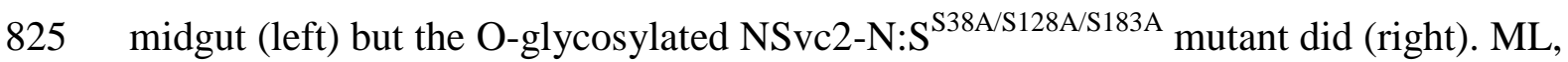

826 midgut lumen; EC, epithelial cell; Bar, $25 \mu \mathrm{m}$. (H) RSV acquisition by SBPHs after

827 pre-feeding with different protein samples. The experiment was repeated three times

828 with 50 SBPHs each group. ${ }^{* *}, p<0.01$ by $t$-test analysis.

830 Fig 4. RSV virion, NSvc2-N and NSvc2-C are co-localized with early or late

831 endosomes, and NSvc2-C separates from RSV virion and NSvc2-N complexes

832 after releasing from endosomes in midgut epithelial cells. (A and B) RSV virion 
833 was detected in early endosomes labeled with the Rab5 antibody (A) or in late

834 endosomes labeled with the Rab7 antibody (B) in midgut epithelial cells.

835 Co-localizations of RSV virion with Rab5 or Rab7 or actin in different endosomes are

836 indicated by arrows. ML, midgut lumen; EC, epithelial cell; Bar, $25 \mu \mathrm{m}$. (C and D)

837 NSvc2-N and NSvc2-C were detected in early endosomes labeled with the EEA1

838 antibody in epithelial cells. Co-localizations of NSvc2-N or NSvc2-C with EEA1 or

839 actin in different endosomes are indicated by arrows. Bar, $25 \mu \mathrm{m}$. (E) RSV virion and

840 NSvc2-N were released from endosome into the cytosol of epithelial cells. The white

841 dashed cycles indicated a region that RSV and NSvc2-N were detected in the cytosol

842 while actin-labeled endosome was absent. Bar, $25 \mu \mathrm{m}$. (F) RSV virion but not

843 NSvc2-C was released from the late endosome in epithelial cells. The white dashed

844 boxes indicated a region that RSV virion was just released from the endosome while

845 NSvc2-C proteins were still associated with actin-labeled endosome. Bar, $25 \mu \mathrm{m}$.

847 Fig 5. NSvc2-C hydrophobic fusion-loop motifs are required for cell-cell

848 membrane fusion. (A) Schematic diagrams showing recombinant NSvc2-N and

849 NSvc2-C. Baculovirus gp64 signal peptide was used to replace the original signal

850 peptide at the N-terminus of NSvc2-N or NSvc2-C. (B) Immunofluorescence labeling

851 of the recombinant NSvc2-N or NSvc2-C in Sf9 cells. Sf9 cells were infected with

852 baculoviruses carrying the recombinant NSvc2-N or NSvc2-C (MOI=5). The infected

853 cells were probed with the NSvc2-N or NSvc2-C specific antibody at $48 \mathrm{~h}$ post

854 infection followed by a TRITC fluorescent-labeled secondary antibody. Bar, $10 \mu \mathrm{m}$. 
855 (C-F) Fusogenic activity assays using Sf9 cells expressing the recombinant NSvc2-N,

856 NSvc2-C or both (NSvc2-N + NSvc2-C). The Sf9 cells were infected with

857 recombinant baculoviruses (MOI=5). After $48 \mathrm{~h}$ post infection, and the growth

858 medium was replaced with a PBS, $\mathrm{pH} 5.0$, for 2 min and then changed back to the

859 normal growth medium for $4 \mathrm{~h}$. Formation of syncytium was observed under a

860 microscope. (G) The number of cells showing syncytium was counted under the

861 microscope. The experiment was repeated three times. ${ }^{* *}, p<0.01$ by the student

$862 t$-test analysis. (H) Sequence alignment using the two fusion loop sequences (FL1 and

863 FL2) from NSvc2-C of five different tenuiviruses (RSV, MSV, RHBV, IWSV and

864 RGSV). Red residues are highly conserved and yellow residues are semi-conserved.

865 Residues indicated with asterisks are hydrophobic residues and were selected for the

866 site-directed mutagenesis. The resulting mutants were later tested for their abilities to

867 induce cell-cell membrane fusion. (I) Two fusion loops (FL1 and FL2, green) were

868 found at the top of the 3D-structure model of NSvc2-C. Three different domains are

869 shown in three different colors, and the boxed region on the 3D-structure is enlarged

870 and shown on the right side. Locations of the hydrophobic residues are displayed. ( $\mathrm{J}$

871 and K) Analyses of the fusogenic activities caused by the WT or mutant NSvc2-C. Sf9

872 cells were infected with recombinant baculoviruses carrying the WT or mutant

873 NSvc2-C for membrane fusion assay $(\mathrm{J})$. The number of cells showing syncytium was

874 recorded and analyzed $(\mathrm{K})$. The experiments were repeated three times. ${ }^{*}, p<0.01$.

876 Fig 6. NSvc2 functions as a molecular bridge to mediate RSV virion entrance into 
SBPH midgut cell. (A) Crude extract of Sf9 cell expressing WT NSvc2,

$878 \mathrm{NSvc} 2^{\mathrm{N} 114 \mathrm{~A} / \mathrm{N} 199 \mathrm{~A} / \mathrm{N} 232 \mathrm{~A}}$ or $\mathrm{NSvc} 2^{\mathrm{F} 460 \mathrm{~A} / \mathrm{F} 489 \mathrm{~A} / \mathrm{Y} 498 \mathrm{~A}}$ was incubated with purified $\mathrm{RSV}$

879 virion for $3 \mathrm{~h}$ at $4{ }^{\circ} \mathrm{C}$, and then used to feed SBPHs for $24 \mathrm{~h}$. Insect midguts were

880 dissected and detected for RSV virion and NSvc2-N using specific antibodies. The

881 boxed regions in the left column were enlarged and shown in the second to fifth

882 columns on the right. The white circled areas show the release of RSV virion (green)

883 and NSvc2-N (red) from endosome into cytosol. The actin labeling signal is shown in

884 blue. ML, midgut lumen; EC, epithelial cell; Bar, $25 \mu \mathrm{m}$. (B) Statistical analysis of

885 RSV infection in SBPH epithelial cells after feeding on the mixtures containing

886 purified RSV virion and the WT or mutant NSvc2-C. Each bar represents three

887 independent biological repeats from each experiment with 50 SBPHs per treatment.

$888 * *, p<0.01$ by student $t$-test analysis.

889

890 Fig 7. Working model for RSV virion entrance into SBPH midgut cells. During

891 SBPH feeding on RSV-infected rice plants, RSV virion, NSvc2-N and NSvc2-C are

892 acquired by the vector and enter insect midgut lumen (Step I). After reaching

893 microvillus surface of midgut lumen, NSvc2-N protein directs the

894 virion:NSvc2-N:NSvc2-C complexes to microvillus surface through recognizing the

895 unidentified microvillus surface receptor(s) (Steps II and III). The complexes-attached

896 midgut lumen membrane undergoes endocytosis and compartmentalizes RSV virion,

897 NSvc2-N and NSvc2-C in vesicles (Steps IV and V). These vesicles further develop

898 into early endosomes (Step VI) and then late endosomes (Step VII). The acidic 
bioRxiv preprint doi: https://doi.org/10.1101/404657; this version posted August 30, 2018. The copyright holder for this preprint (which was not certified by peer review) is the author/funder, who has granted bioRxiv a license to display the preprint in perpetuity. It is made available under aCC-BY 4.0 International license.

899 condition inside the late endosomes triggers NSvc2-C to induce cell-cell membrane

900 fusion (Step VII). Finally, RSV virion:NSvc2-N complexes are released from the late

901 endosomes into the cytosol of epithelial cells for further RSV spread in midgut cells

902 (Step VIII). 


\section{Supporting information}

904 S1 Fig. Schematic diagram showing the full length NSvc2 (A) and the

905 recombinant soluble NSvc2-N (NSvc2-N:S) (B). Locations of the putative signal

906 peptide, hydrophobic domains, and glycosylation sites are indicated. The signal

907 peptide of NSvc2-N:S is replaced with the signal peptide of Gp64.

908

S2 Fig. The effects of pre-incubation with purified NSvc2-N:S (A), TSWV Gn:S

910 (B) and sucrose alone (C) on RSV entrance into SBPH midguts. The boxed

911 regions are enlarged and shown on the right side. The overlapping fluorescence

912 spectra were from the white dashed line indicated areas (right top and bottom panels).

913 EC, epithelial cell; Bar, $25 \mu \mathrm{m}$.

914

915 S3 Fig. Time course study of NSvc2-C subcellular localization during RSV

916 entrance into SBPH midgut. (A) NSvc2-C was co-localized with RSV virion on

917 midgut microvillus surface (blue) at $4 \mathrm{~h}$ post feeding on RSV-infected seedlings. The

918 boxed region was enlarged and shown on the right side. The labeled NSvc2-C is

919 shown in red and the labeled RSV virion is shown in green. (B) NSvc2-C and RSV

920 virion were co-localized in the endosomal-like vesicles in midgut epithelial cells at 8

$921 \mathrm{~h}$ post infestation. (C) RSV virion was detected in cytoplasm of epithelial cells at $16 \mathrm{~h}$

922 post infestation but not NSvc2-C. (D) NSvc2-C was again not detected together with

923 RSV virion at $24 \mathrm{~h}$ post infestation. (E-H) Analyses of overlapping fluorescence

924 spectra from the white dashed lines indicated regions in the merged images. The 

lumen; EC, epithelial cell; Bar, $10 \mu \mathrm{m}$.

927

S4 Fig. NSvc2-C hydrophobic fusion-loop motifs are required for cell-cell membrane fusion. (A) A diagram showing different NSvc2-C domains. Different domains are shown in yellow (domain I), red (domain II) and blue color (domain III).

931 Fusion loops (FL1 and FL2) are shown in green, and a hydrophobic region in gray. (B)

932 A three dimensional homology based model of NSvc2-C with the same color 933 arrangement as shown in (A). (C) Schematic representations of the wild type and FL 934 deletion NSvc2-C mutants. Locations of FL1 (black) and FL2 (gray) are shown. 935 Deletions of one or double FLs are indicated with upward open arrows. (D) 936 Expressions of FLAG-tagged WT or mutant NSvc2-C in Sf9 cells were determined by 937 immunoblotting. The blots were probed with a FLAG-specific antibody. Arrows 938 indicate the bands of the expressed NSvc2-C proteins. ( $\mathrm{E}$ and F) Analyses of 939 fusogenic activities of the WT or mutant NSvc2-C. Sf9 cells were infected with 940 recombinant baculoviruses carrying the WT or deletion mutants. At $48 \mathrm{~h}$ post 941 infection, the cells were treated with PBS, pH5.0, for the cell-cell membrane fusion 942 assays (E). The numbers of cells showing syncytium were recorded and analyzed (F).

943 The experiment was repeated three times. $* *, p<0.01$. 

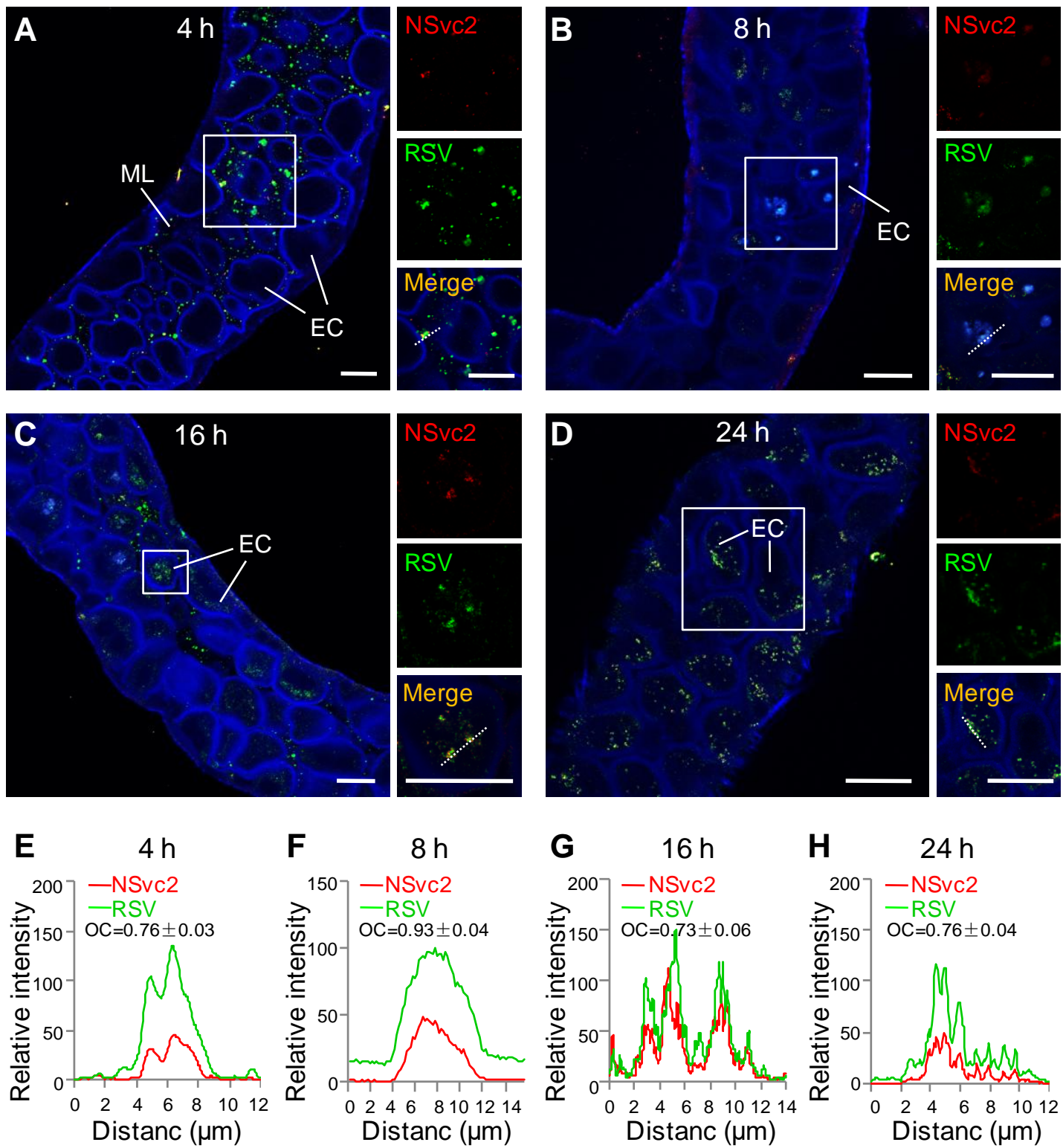

\section{$948 \quad$ Fig 1.}


A

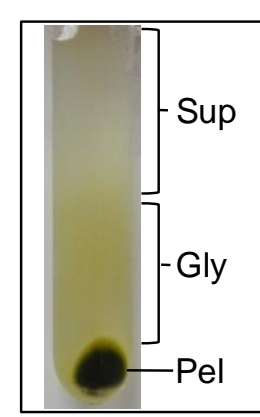

B

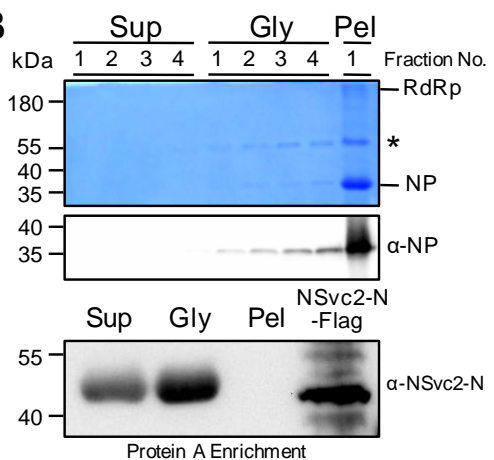

C

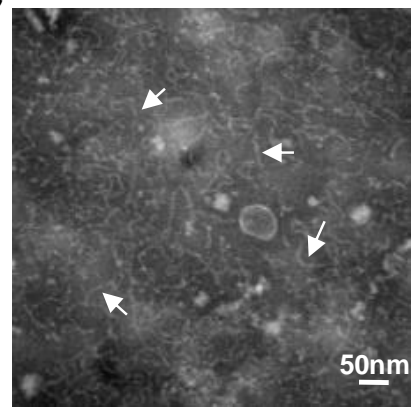

D NSvc2

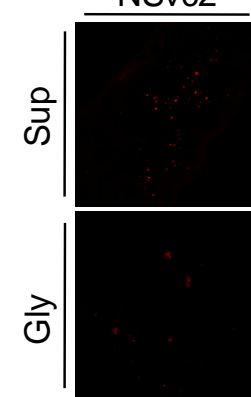

$\bar{\omega}$
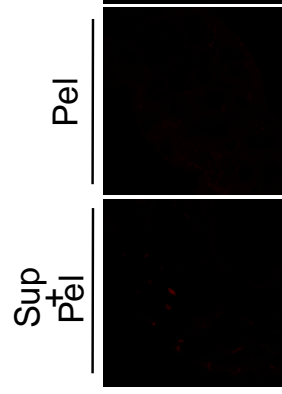

$\mathbf{E}$
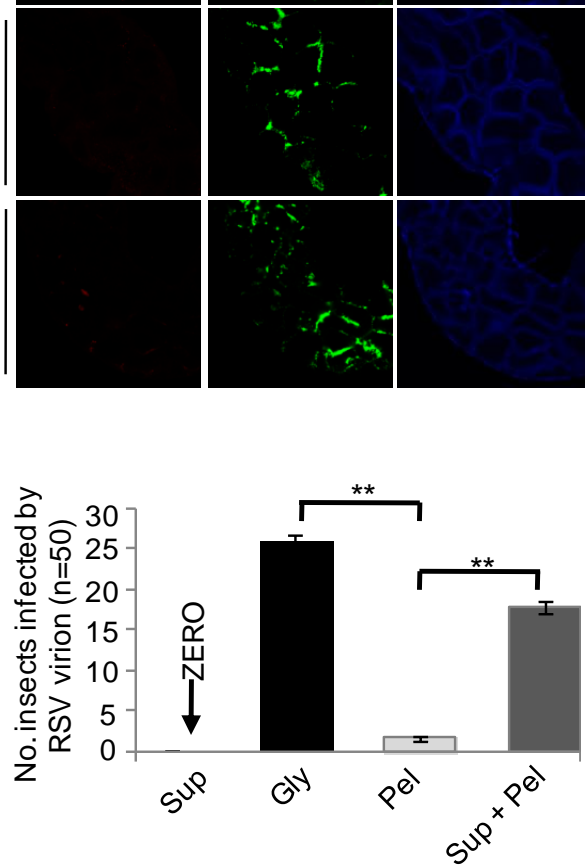

Merge
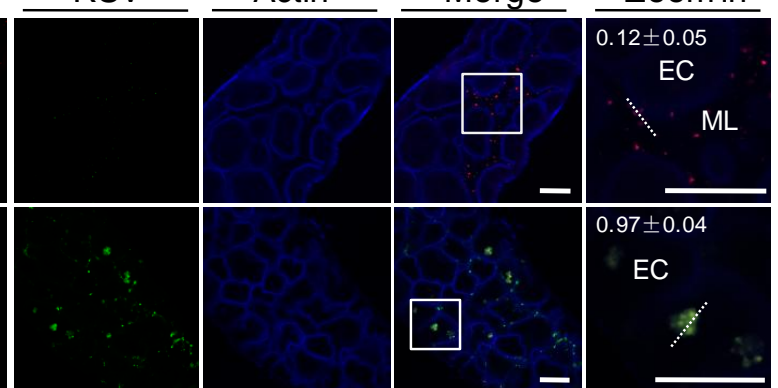

EC
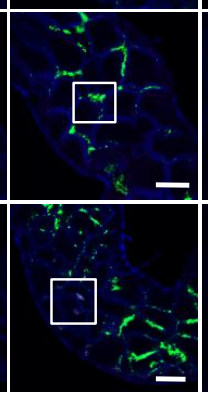

$\mathbf{F}$

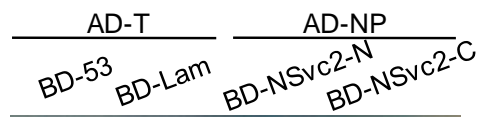

EC
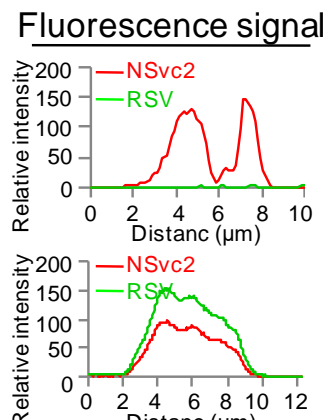

(1)

200-NSvc2

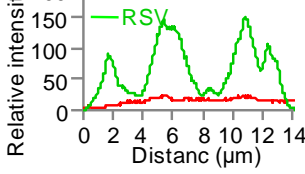

EC

$0.95 \pm 0.03$

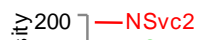

क्. $150-$-RSV

离 100

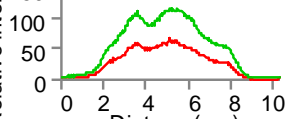

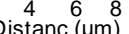

SD / -Trp / -Leu / -His / -Ade

$951 \quad$ Fig 2. 
A NSvc2-N:S B
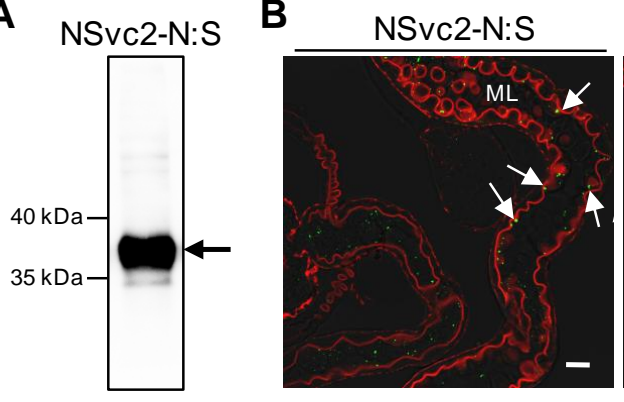

D

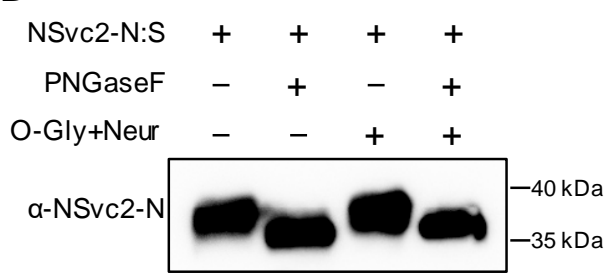

$\mathbf{E}$

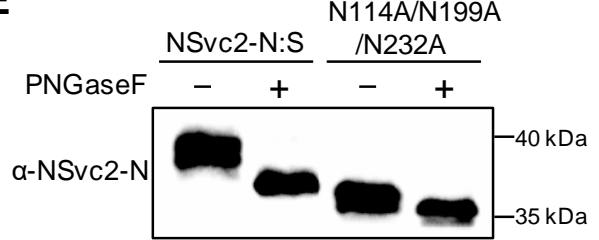

$\mathbf{F}$

953

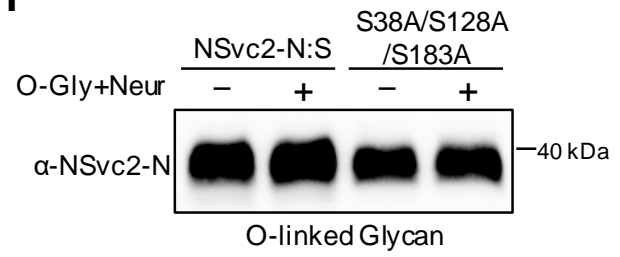

TSWV Gn:S

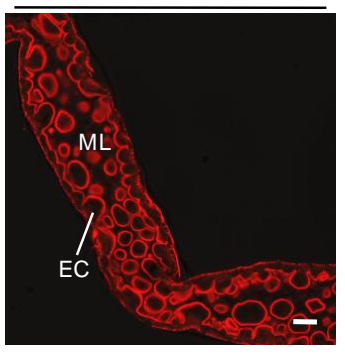

G

NSvc2-N:S N114AN199AN232A

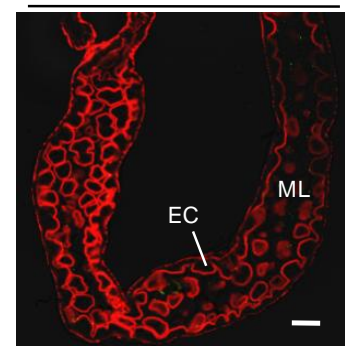

NSvc2-N:SS38A/S128A/S183A

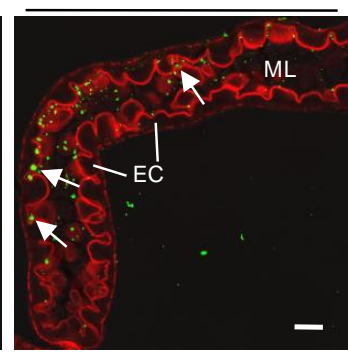

H

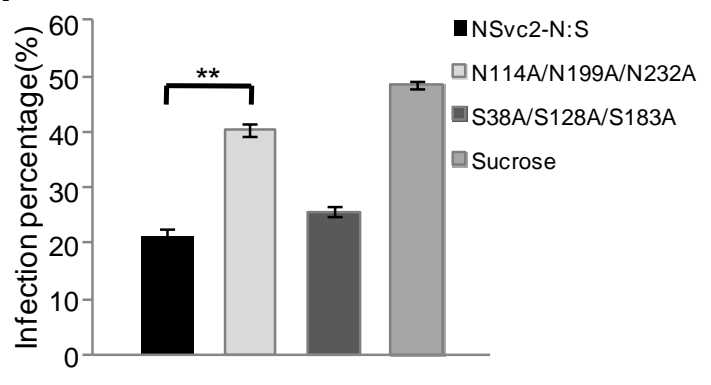

\section{$954 \quad$ Fig 3.}




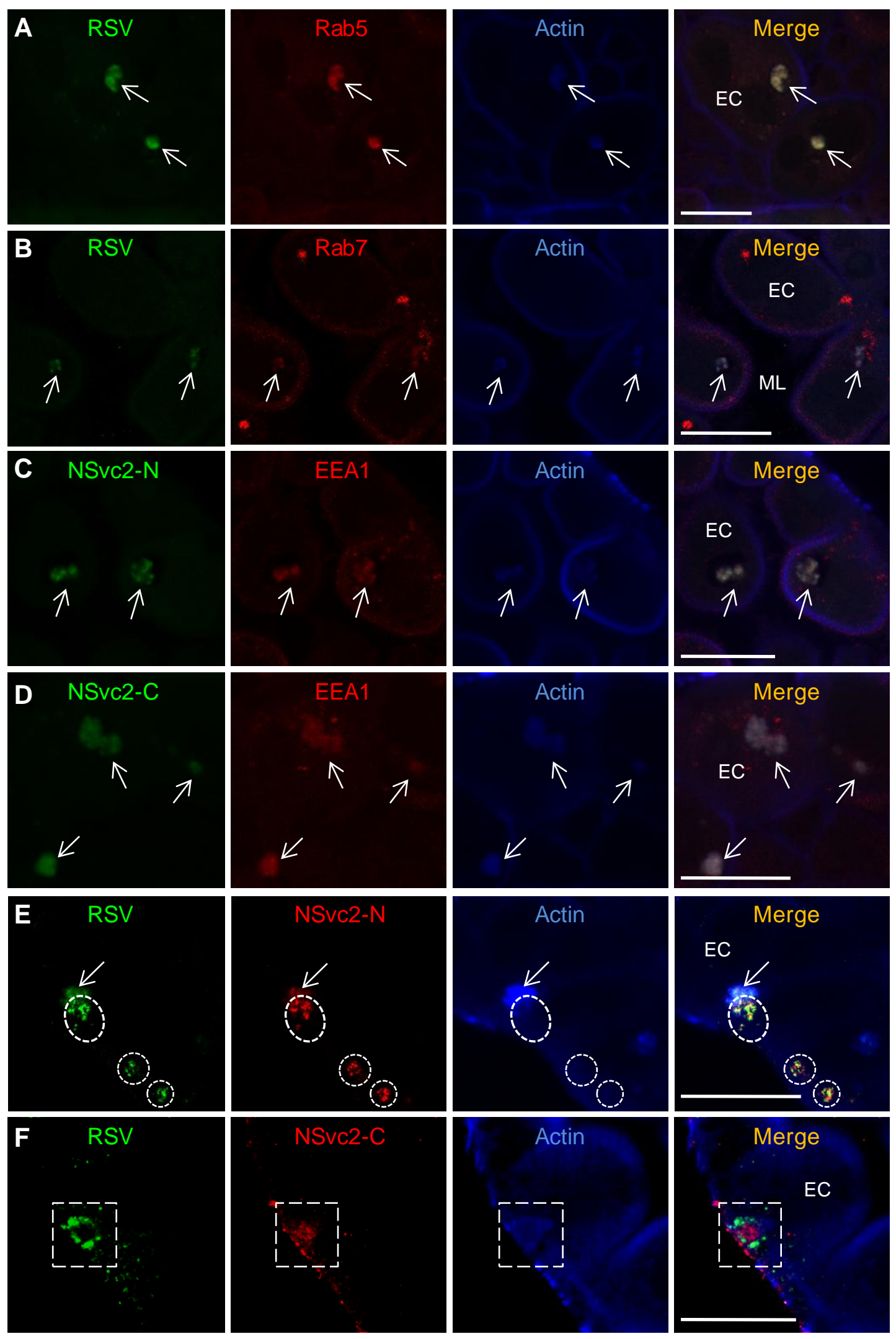

Fig 4. 
A

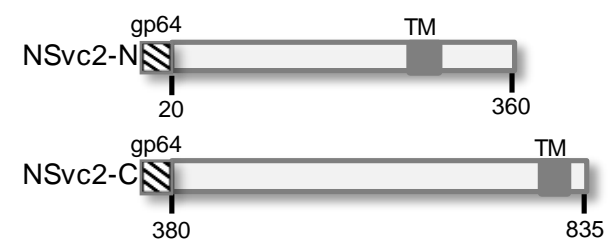

B

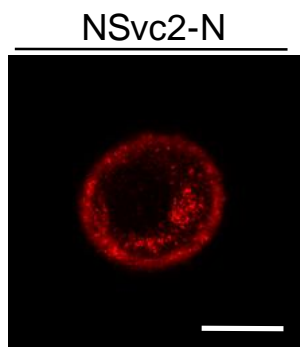

G

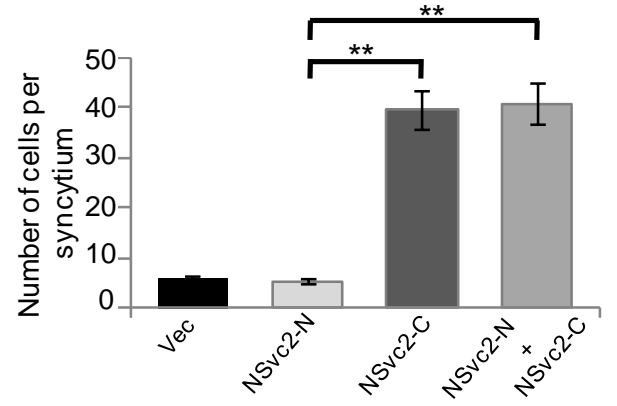

H

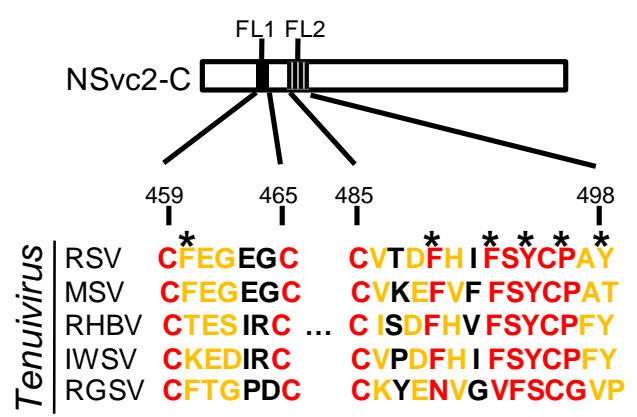

$\mathbf{J}$

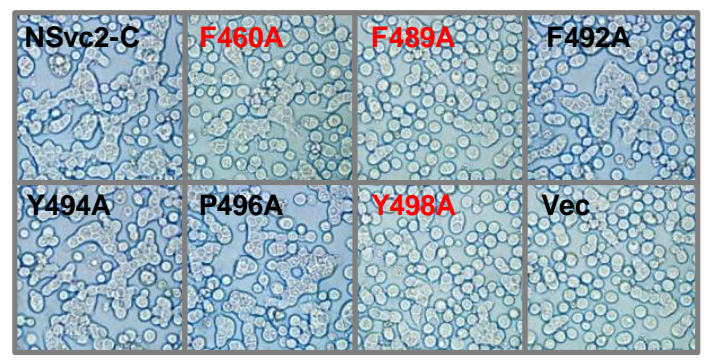

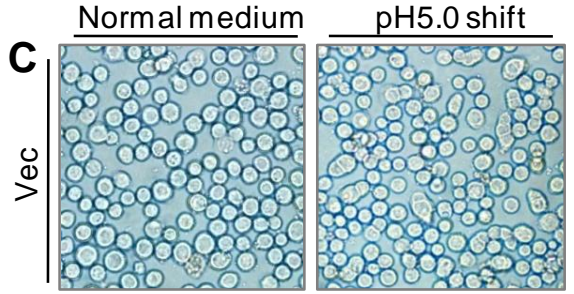

D 800005800 प:
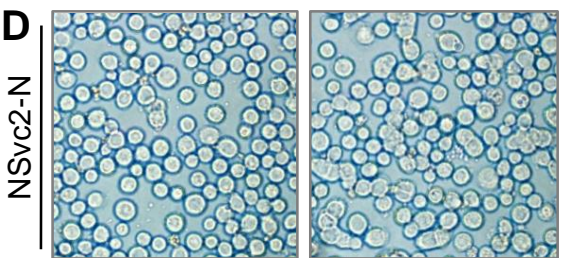

E

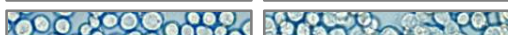

O

ऽ)

2

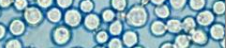

(380
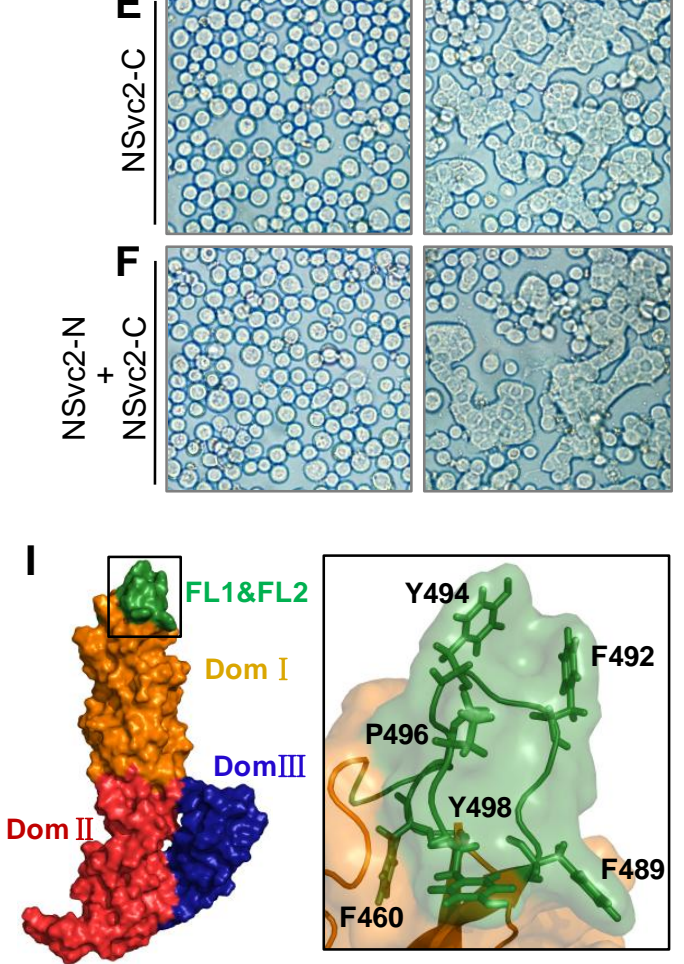

K

-NSvc2-C $\square$ F460A $\square$ F489A $\square$ F492A

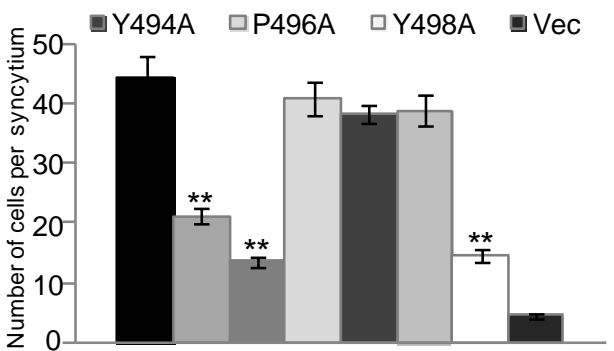

\section{$960 \quad$ Fig 5.}


bioRxiv preprint doi: $h t t p s: / / d o i$ org/101101/404657; this version posted August 30, 2018. The copyright holder for this preprint (which was not certified by peer review) is the author/funder, who has granted bioRxiv a license to display the preprint in perpetuity. It is made available under aCC-BY 4.0 International license.

962

A RSV NSvc2-N Actin Merge

\section{B}

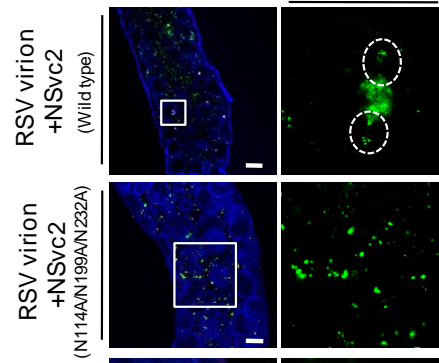

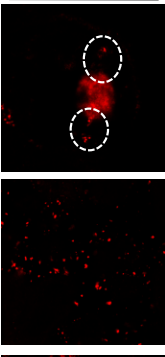
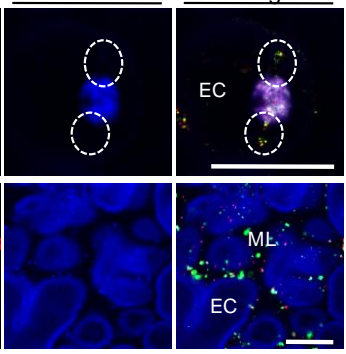

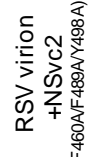
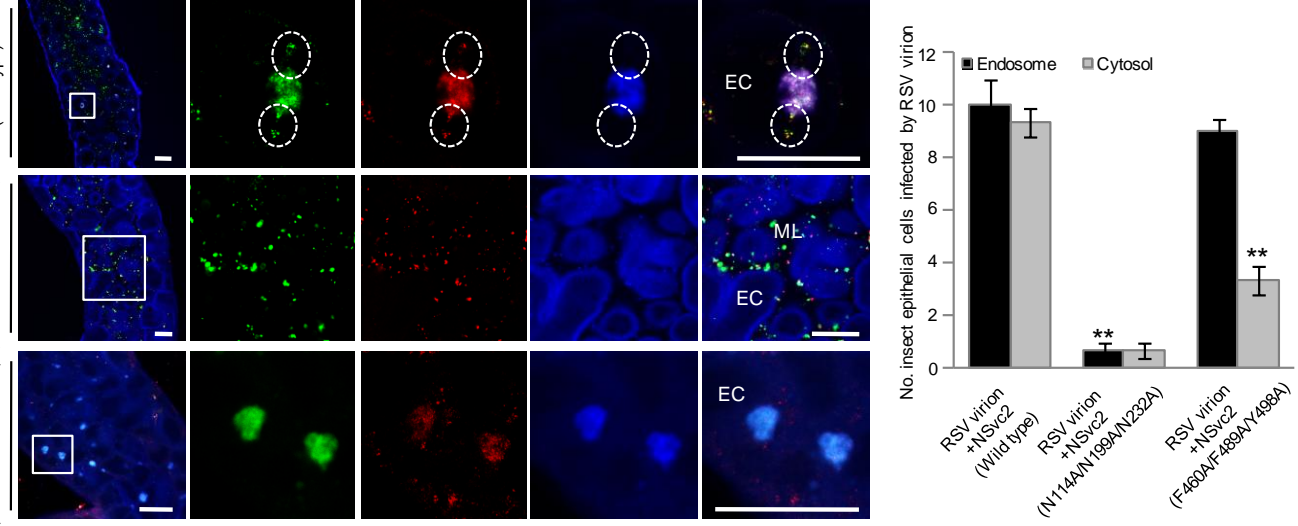

\section{$963 \quad$ Fig 6.}

964 


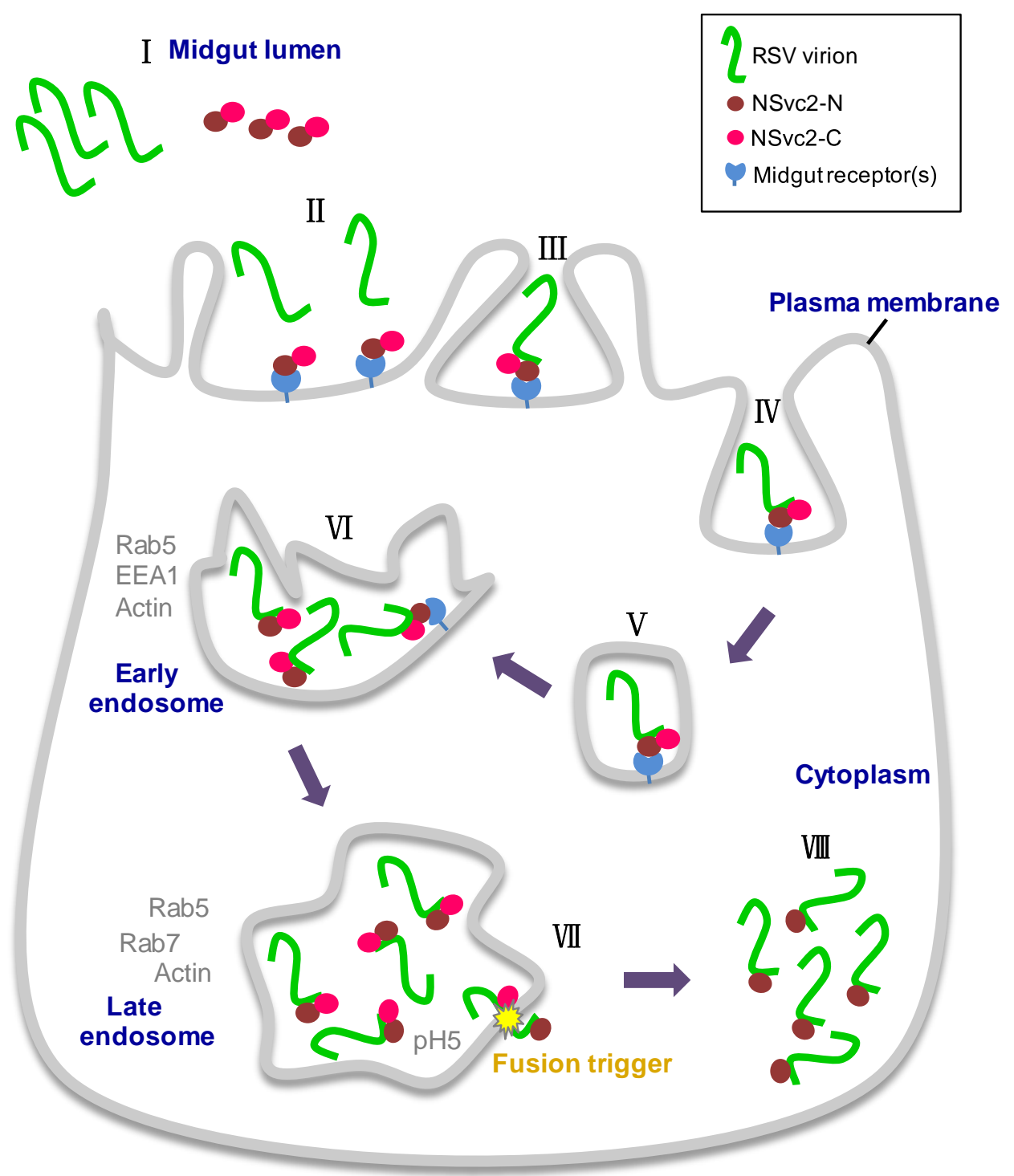

965

$966 \quad$ Fig 7.

967 
A

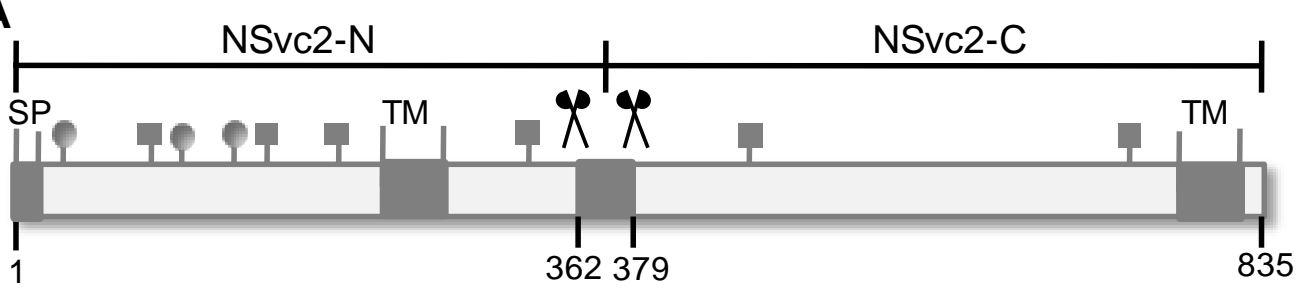

B

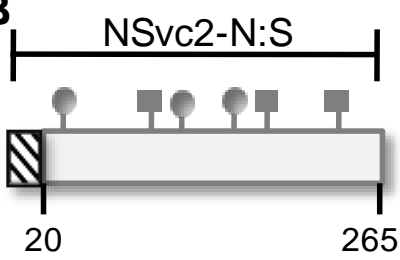

N-linked glycan sites

O-linked glycan sites

Hydrophobic domains

$\mathbb{N}$ Gp64 signal peptide

\section{S1 Fig.}

970 

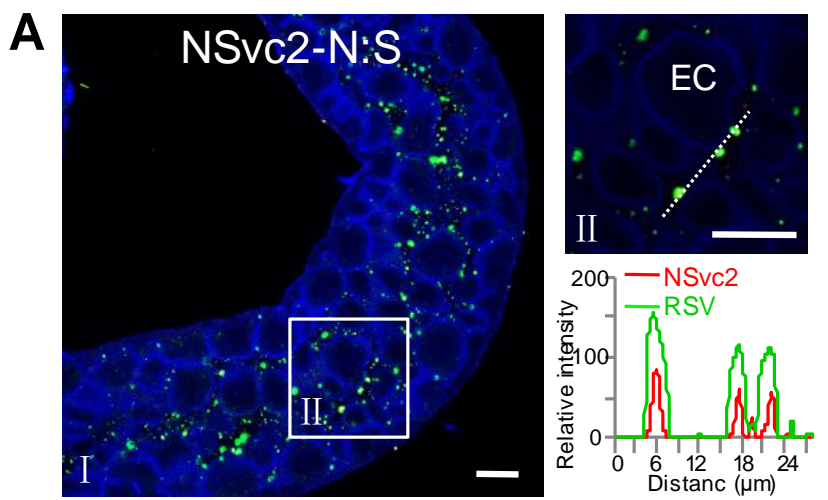

B

\section{TSWV Gn:S}
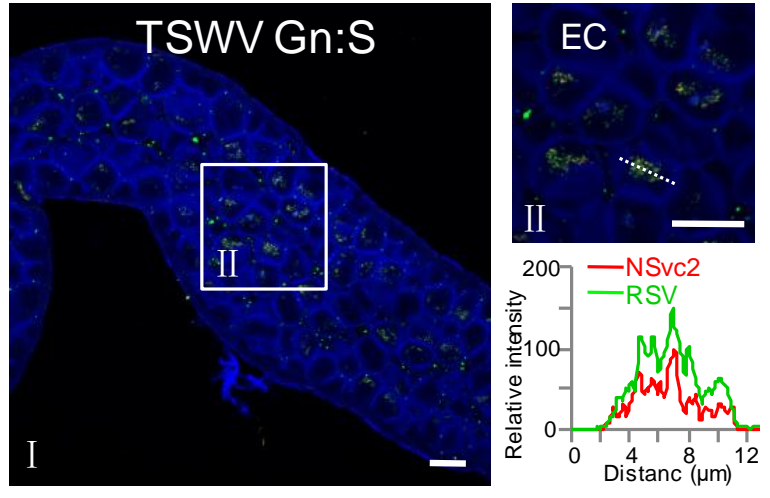

C
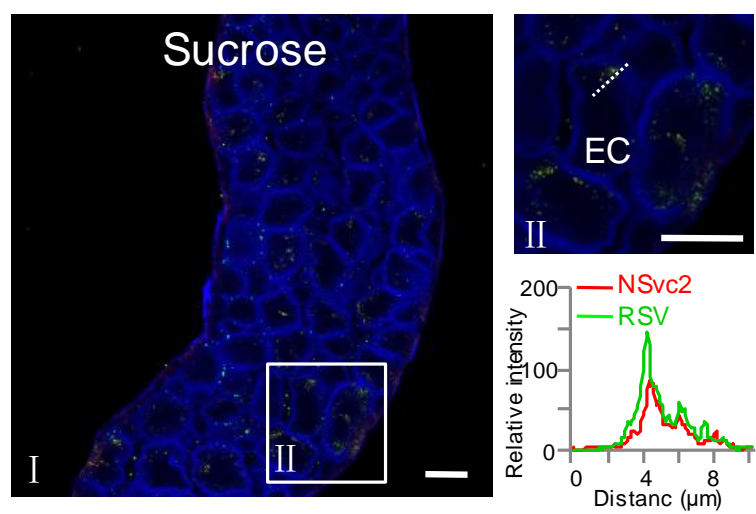

972 S2 Fig. 

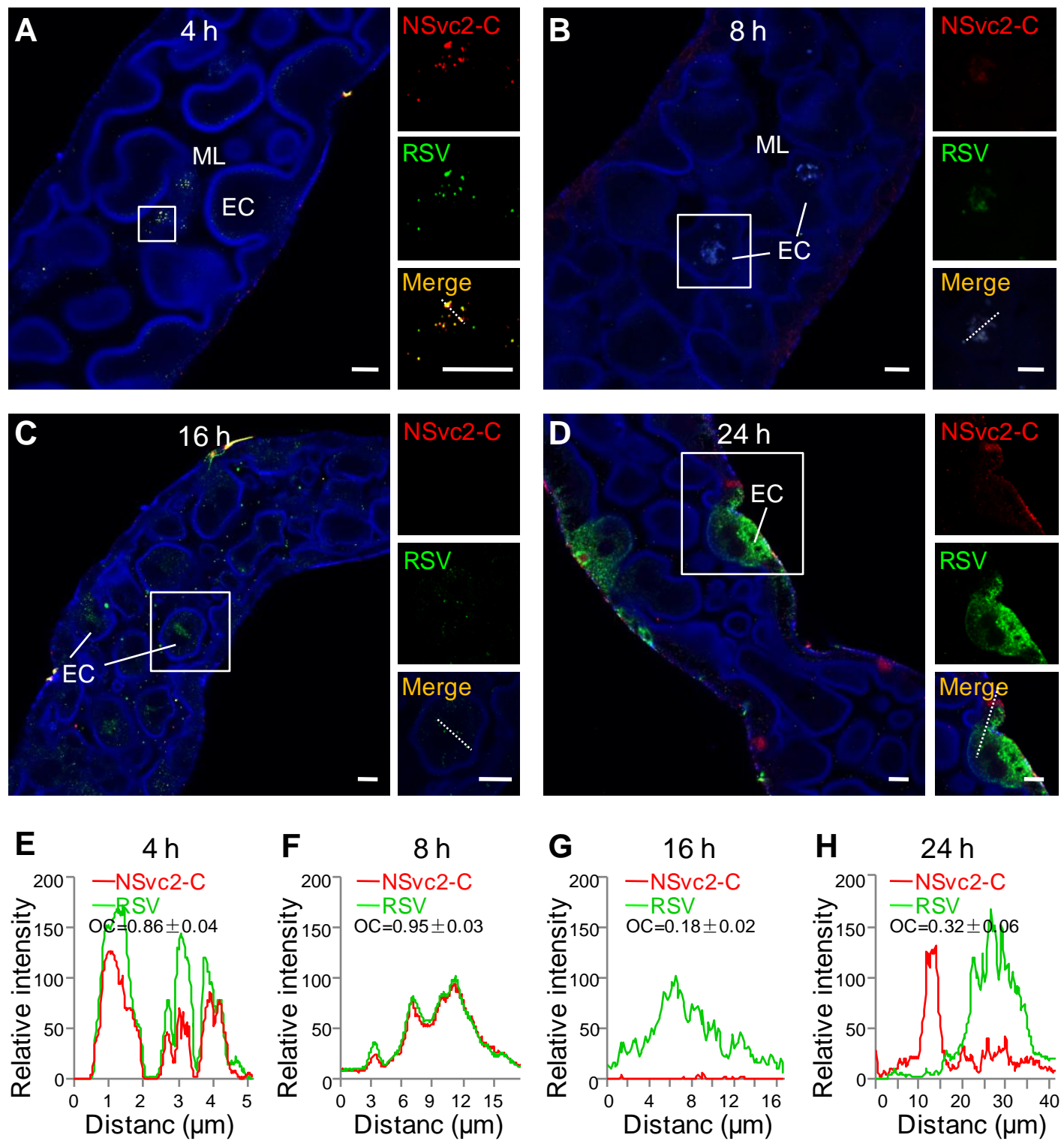

S3 Fig. 

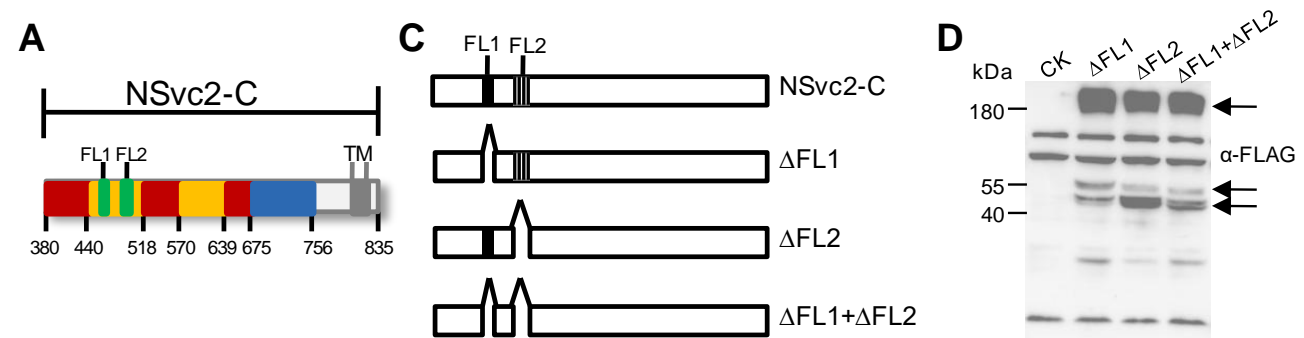

B

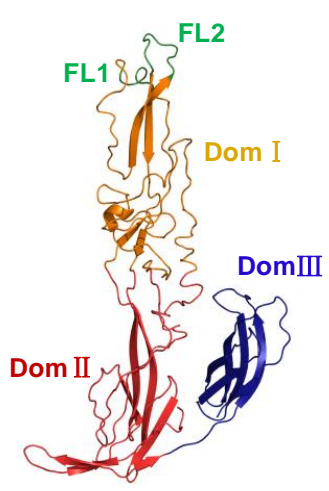

E

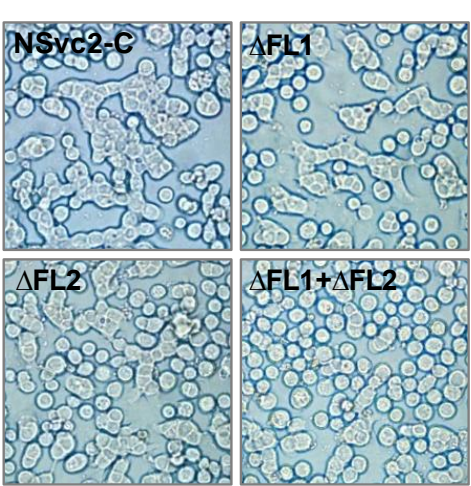

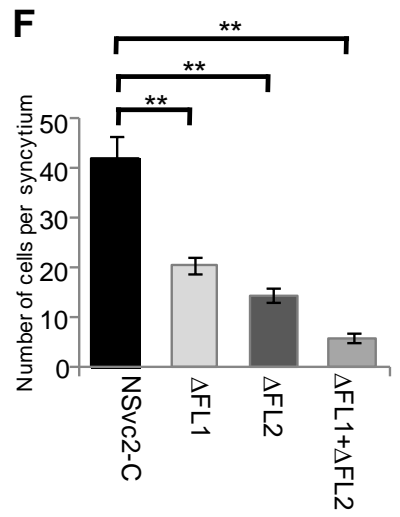

977

978 S4 Fig.

979 
$980 \quad$ S1 Table.

\begin{tabular}{|c|c|c|c|c|}
\hline Clone & \multicolumn{2}{|c|}{ Primer } & Primer sequence (from 5' to $3^{\prime}$ ) & Purpose \\
\hline \multirow{4}{*}{$\begin{array}{l}\text { pFastbac1-GP64 } \\
\text {-6xhis-NSvc2-N:S } \\
\text {-6xhis }\end{array}$} & $\mathrm{F}$ & P3209 & $\begin{array}{c}\text { CCggatccATGCACCACCACCACCACCACCCAAT } \\
\underline{\text { ACCTTTCCCTGACAC }}\end{array}$ & \multirow{4}{*}{$\begin{array}{c}\text { To amplify RSV } \\
\text { NSvc2-N:S } \\
\text { fragment (20-265aa) } \\
\text { and GP64 fragment, } \\
\text { then clone into } \\
\text { pFastbac1 }\end{array}$} \\
\hline & $\mathrm{R}$ & P3210 & $\begin{array}{c}\text { CCGctcgagTCAGTGGTGGTGGTGGTGGTGTGAAT } \\
\underline{\text { ATCTATAGATGTGAG }}\end{array}$ & \\
\hline & $\mathrm{F}$ & P3253 & CGggatccATGGTAAGCGCTATTGTTTT & \\
\hline & $\mathrm{R}$ & P3254 & GAagatctGTTGCAGTGCTCCGCCGCAA & \\
\hline \multirow{4}{*}{$\begin{array}{l}\text { pFastbac1-GP64 } \\
\text {-6xhis-TSWV-Gn:S } \\
\text {-6xhis }\end{array}$} & $\mathrm{F}$ & P3391 & $\begin{array}{c}\text { CCggatccATGCACCACCACCACCACCACGCTAA } \\
\underline{\text { AGTAGAAATAATTCG }}\end{array}$ & \multirow{4}{*}{$\begin{array}{c}\text { To amplify TSWV } \\
\text { Gn:S fragment } \\
\text { (35-300aa) and } \\
\text { GP64 fragment, } \\
\text { then clone into } \\
\text { pFastbac1 }\end{array}$} \\
\hline & $\mathrm{R}$ & P3392 & $\begin{array}{c}\text { CCGctcgagTCAGTGGTGGTGGTGGTGGTGTCTGA } \\
\text { CTCTTTTGTTTGTTT } \\
\end{array}$ & \\
\hline & $\mathrm{F}$ & P3253 & CGggatccATGGTAAGCGCTATTGTTTT & \\
\hline & $\mathrm{R}$ & P3254 & GAagatctGTTGCAGTGCTCCGCCGCAA & \\
\hline \multirow{4}{*}{$\begin{array}{l}\text { pFastbac1-GP64- } \\
\text { NSvc2-N-1xFlag }\end{array}$} & $\mathrm{F}$ & LG-1 & CGGAGCACTGCAACAGATCCatggcaccaatacctttccc & \multirow{4}{*}{$\begin{array}{l}\text { To amplify two } \\
\text { fragments and do } \\
\text { homologous } \\
\text { recombination to } \\
\text { obtain plasmid }\end{array}$} \\
\hline & $\mathrm{R}$ & LG-2 & gggaaaggtattggtgccatGGATCTGTTGCAGTGCTCCG & \\
\hline & $\mathrm{F}$ & LG-3 & $\begin{array}{c}\text { agggtaggaaagtagagaatGATTACAAGGATGATGATG } \\
\text { A }\end{array}$ & \\
\hline & $\mathrm{R}$ & LG-4 & TCATCATCATCCTTGTAATCattctctactttcetaccet & \\
\hline \multirow{4}{*}{$\begin{array}{l}\text { pFastbac1-GP64- } \\
\text { NSvc2-C-1xFlag }\end{array}$} & $\mathrm{F}$ & $\mathrm{P} 4500$ & CGggatccATGGAATCCTGTCAAGACCTTG & \multirow{4}{*}{$\begin{array}{c}\text { To amplify RSV } \\
\text { NSvc2-C and GP64 } \\
\text { fragments, then } \\
\text { clone into } \\
\text { pFastbac1 }\end{array}$} \\
\hline & $\mathrm{R}$ & P4501 & $\begin{array}{c}\text { CGctcgagTTACTTATCATCATCATCCTTGTAATC } \underline{\text { AT }} \\
\underline{\text { CAACCTGTCTAATGTC }}\end{array}$ & \\
\hline & $\mathrm{F}$ & P3253 & CGggatccATGGTAAGCGCTATTGTTTT & \\
\hline & $\mathrm{R}$ & P3254 & GAagatctGTTGCAGTGCTCCGCCGCAA & \\
\hline \multirow{4}{*}{$\begin{array}{l}\text { pFastbac1-GP64- } \\
\text { NSvc2-C( } \Delta \text { FL1) } \\
\text {-1xFlag }\end{array}$} & $\mathrm{F}$ & P4726 & CCACCATCGGGCGCGGATCCatggtaagcgctattgtttt & \multirow{4}{*}{$\begin{array}{l}\text { To amplify deletion } \\
\text { mutant fragment } \\
\text { and vector } \\
\text { fragment, then do } \\
\text { homologous } \\
\text { recombination to } \\
\text { obtain plasmid }\end{array}$} \\
\hline & $\mathrm{R}$ & P4727 & aaaacaatagcgcttaccatGGATCCGCGCCCGATGGTGG & \\
\hline & $\mathrm{F}$ & $\mathrm{P} 4563$ & agagaggaatggaaaAGAACTGATGGAACA & \\
\hline & $\mathrm{R}$ & P4564 & tgttccatcagttctTTTCCATTCСТCТCT & \\
\hline \multirow{4}{*}{$\begin{array}{l}\text { pFastbac1-GP64- } \\
\text { NSvc2-C( } \Delta \text { FL2) } \\
\text {-1xFlag }\end{array}$} & $\mathrm{F}$ & P3253 & CGggatccATGGTAAGCGCTATTGTTTT & \multirow{4}{*}{$\begin{array}{c}\text { To amplify } \\
\text { NSvc2-C deletion } \\
\text { mutant } \\
\text { fragments, then mix } \\
\text { the two fragments } \\
\text { and do overlap PCR } \\
\text { to obtain the mutant } \\
\text { and clone into } \\
\text { pFastbac1 }\end{array}$} \\
\hline & $\mathrm{R}$ & $\mathrm{P} 4565$ & ctgagctttgattatCACTACAATTGGAAG & \\
\hline & $\mathrm{F}$ & P4566 & cttccaattgtagtgATAATCAAAGCTCAG & \\
\hline & $\mathrm{R}$ & $\mathrm{P} 4501$ & $\begin{array}{c}\text { CGctcgagTTACTTATCATCATCATCCTTGTAATCAT } \\
\underline{\text { CAACCTGTCTAATGTC }}\end{array}$ & \\
\hline
\end{tabular}




\begin{tabular}{|c|c|c|c|c|}
\hline \multirow{4}{*}{$\begin{array}{l}\text { pFastbac1-GP64- } \\
\text { NSve2-C( } \Delta \text { FL1 } \\
\& \Delta \text { FL2)-1xFlag }\end{array}$} & $\mathrm{F}$ & $\mathrm{P} 4726$ & CCACCATCGGGCGCGGATCCatggtaagcgctattgtttt & \multirow{4}{*}{$\begin{array}{l}\text { To amplify deletion } \\
\text { mutant fragment } \\
\text { and vector } \\
\text { fragment, then do } \\
\text { homologous } \\
\text { recombination to } \\
\text { obtain plasmid }\end{array}$} \\
\hline & $\mathrm{R}$ & P4727 & aaaacaatagcgcttaccatGGATCCGCGCCCGATGGTGG & \\
\hline & $\mathrm{F}$ & P4565 & ctgagctttgattatCACTACAATTGGAAG & \\
\hline & $\mathrm{R}$ & P4566 & cttccaattgtagtgATAATCAAAGCTCAG & \\
\hline \multirow{4}{*}{$\begin{array}{l}\text { pFastbac1-GP64- } \\
\text { NSvc2-C(F460A) } \\
\text {-1xFlag }\end{array}$} & $\mathrm{F}$ & P4726 & CCACCATCGGGCGCGGATCCatggtaagcgctattgtttt & \multirow{4}{*}{$\begin{array}{l}\text { To amplify point } \\
\text { mutant fragment } \\
\text { and vector } \\
\text { fragment, then do } \\
\text { homologous } \\
\text { recombination to } \\
\text { obtain plasmid }\end{array}$} \\
\hline & $\mathrm{R}$ & $\mathrm{P} 4727$ & aaaacaatagcgcttaccatGGATCCGCGCCCGATGGTGG & \\
\hline & $\mathrm{F}$ & $\mathrm{P} 2603$ & GAGGAATGGAAATGT $g c t$ GAAGGGGAAGGTTGC & \\
\hline & $\mathrm{R}$ & P2604 & GCAACСТTCСССТTCagcACATTTCCATTCCTC & \\
\hline \multirow{4}{*}{$\begin{array}{l}\text { pFastbac1-GP64- } \\
\text { NSvc2-C(F489A) } \\
\text {-1xFlag }\end{array}$} & $\mathrm{F}$ & $\mathrm{P} 4726$ & CCACCATCGGGCGCGGATCCatggtaagcgctattgtttt & \multirow{4}{*}{$\begin{array}{l}\text { To amplify point } \\
\text { mutant fragment } \\
\text { and vector } \\
\text { fragment, then do } \\
\text { homologous } \\
\text { recombination to } \\
\text { obtain plasmid }\end{array}$} \\
\hline & $\mathrm{R}$ & P4727 & aaaacaatagcgcttaccatGGATCCGCGCCCGATGGTGG & \\
\hline & $\mathrm{F}$ & $\mathrm{P} 2605$ & TATTGTGTCACTGATgccCACATATTTTCCTAT & \\
\hline & $\mathrm{R}$ & $\mathrm{P} 2606$ & ATAGGAAAATATGTG $g g c$ ATCAGTGACACAATA & \\
\hline \multirow{4}{*}{$\begin{array}{l}\text { pFastbac1-GP64- } \\
\text { NSvc2-C(F492A) } \\
\text {-1xFlag }\end{array}$} & $\mathrm{F}$ & $\mathrm{P} 4726$ & CCACCATCGGGCGCGGATCCatggtaagcgctattgtttt & \multirow{4}{*}{$\begin{array}{l}\text { To amplify point } \\
\text { mutant fragment } \\
\text { and vector } \\
\text { fragment, then do } \\
\text { homologous } \\
\text { recombination to } \\
\text { obtain plasmid }\end{array}$} \\
\hline & $\mathrm{R}$ & P4727 & aaaacaatagcgcttaccatGGATCCGCGCCCGATGGTGG & \\
\hline & $\mathrm{F}$ & P2607 & $\begin{array}{c}\text { GTCACTGATTTCCACGCAgctTCCTATTGCCCAG } \\
\text { CA }\end{array}$ & \\
\hline & $\mathrm{R}$ & P2608 & $\begin{array}{l}\text { TGCTGGGCAATAGGA } a g c \text { TGCGTGGAAATCAGT } \\
\text { GAC }\end{array}$ & \\
\hline \multirow{4}{*}{$\begin{array}{l}\text { pFastbac1-GP64- } \\
\text { NSvc2-C(F494A) } \\
\text {-1xFlag }\end{array}$} & $\mathrm{F}$ & P4726 & CCACCATCGGGCGCGGATCCatggtaagcgctattgtttt & \multirow{4}{*}{$\begin{array}{l}\text { To amplify point } \\
\text { mutant fragment } \\
\text { and vector } \\
\text { fragment, then do } \\
\text { homologous } \\
\text { recombination to } \\
\text { obtain plasmid }\end{array}$} \\
\hline & $\mathrm{R}$ & $\mathrm{P} 4727$ & aaaacaatagcgcttaccatGGATCCGCGCCCGATGGTGG & \\
\hline & $\mathrm{F}$ & P2609 & TTCCACATATTTTCC $g c t$ TGCCCAGCATACCAC & \\
\hline & $\mathrm{R}$ & $\mathrm{P} 2610$ & GTGGTATGCTGGGCA $a g c$ GGAAAATATGTGGAA & \\
\hline \multirow{4}{*}{$\begin{array}{l}\text { pFastbac1-GP64- } \\
\text { NSvc2-C(F496A) } \\
\text {-1xFlag }\end{array}$} & $\mathrm{F}$ & P4726 & CCACCATCGGGCGCGGATCCatggtaagcgctattgtttt & \multirow{4}{*}{$\begin{array}{l}\text { To amplify point } \\
\text { mutant fragment } \\
\text { and vector } \\
\text { fragment, then do } \\
\text { homologous } \\
\text { recombination to } \\
\text { obtain plasmid }\end{array}$} \\
\hline & $\mathrm{R}$ & P4727 & aaaacaatagcgcttaccatGGATCCGCGCCCGATGGTGG & \\
\hline & $\mathrm{F}$ & $\mathrm{P} 2611$ & ATATTTTCCTATTGC $g c a$ GCATACCACTACAAT & \\
\hline & $\mathrm{R}$ & $\mathrm{P} 2612$ & ATTGTAGTGGTATGCtgcGCAATAGGAAAATAT & \\
\hline
\end{tabular}




\begin{tabular}{|c|c|c|c|c|}
\hline \multirow{4}{*}{$\begin{array}{c}\text { pFastbac1-GP64- } \\
\text { NSvc2-C(F498A) } \\
\text {-1xFlag }\end{array}$} & $\mathrm{F}$ & $\mathrm{P} 4726$ & CCACCATCGGGCGCGGATCCatggtaagcgctattgtttt & \multirow{4}{*}{$\begin{array}{l}\text { To amplify point } \\
\text { mutant fragment } \\
\text { and vector } \\
\text { fragment, then do } \\
\text { homologous } \\
\text { recombination to } \\
\text { obtain plasmid }\end{array}$} \\
\hline & $\mathrm{R}$ & $\mathrm{P} 4727$ & aaaacaatagcgcttaccatGGATCCGCGCCCGATGGTGG & \\
\hline & $\mathrm{F}$ & $\mathrm{P} 2613$ & TCCTATTGCCCAGCA $g c c$ CACTACAATTGGAAG & \\
\hline & $\mathrm{R}$ & $\mathrm{P} 2614$ & CTTCCAATTGTAGTGggctGCTGGGCAATAGGA & \\
\hline \multirow{8}{*}{$\begin{array}{c}\text { pFastbac1-GP64 } \\
\text {-6xHis-NSvc2-N } \\
\text { (N114A/N199A/N232)- } \\
\text { 6xHis }\end{array}$} & $\mathrm{F}$ & P4726 & CCACCATCGGGCGCGGATCCatggtaagcgctattgtttt & \multirow{8}{*}{$\begin{array}{l}\text { To amplify point } \\
\text { mutant fragment } \\
\text { and vector } \\
\text { fragment, then do } \\
\text { homologous } \\
\text { recombination to } \\
\text { obtain plasmid }\end{array}$} \\
\hline & $\mathrm{R}$ & $\mathrm{P} 4727$ & aaaacaatagcgcttaccatGGATCCGCGCCCGATGGTGG & \\
\hline & $\mathrm{F}$ & LG-5 & GATGCTATTCTGCAGgctGTATCTCTGGTTGGC & \\
\hline & $\mathrm{R}$ & LG-6 & GCCAACCAGAGATACagcCTGCAGAATAGCATC & \\
\hline & $\mathrm{F}$ & LG-7 & TCTGCATGTGAGGTCgctGTGAGTGACCAGACA & \\
\hline & $\mathrm{R}$ & LG-8 & TGTCTGGTCACTCACagcGACCTCACATGCAGA & \\
\hline & $\mathrm{F}$ & LG-9 & AAGGGTGGTTGTCAA $g c t$ GTTACTTGTCACCCA & \\
\hline & $\mathrm{R}$ & LG-10 & TGGGTGACAAGTAACagcTTGACAACCACCCTT & \\
\hline \multirow{8}{*}{$\begin{array}{c}\text { pFastbac1-GP64 } \\
\text {-6xHis-NSvc2-N } \\
\text { (S38A/S128A/S183A)- } \\
\text { 6xHis }\end{array}$} & $\mathrm{F}$ & P4726 & CCACCATCGGGCGCGGATCCatggtaagcgctattgtttt & \multirow{8}{*}{$\begin{array}{l}\text { To amplify point } \\
\text { mutant fragment } \\
\text { and vector } \\
\text { fragment, then do } \\
\text { homologous } \\
\text { recombination to } \\
\text { obtain plasmid }\end{array}$} \\
\hline & $\mathrm{R}$ & $\mathrm{P} 4727$ & aaaacaatagcgcttaccatGGATCCGCGCCCGATGGTGG & \\
\hline & $\mathrm{F}$ & LG-11 & AGGGAGAGAGTTCCAgctGAGATTGTCAAGGTC & \\
\hline & $\mathrm{R}$ & LG-12 & GACCTTGACAATCTCagcTGGAACTCTCTCCCT & \\
\hline & $\mathrm{F}$ & LG-13 & 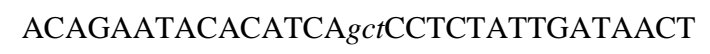 & \\
\hline & $\mathrm{R}$ & LG-14 & AGTTATCAATAGAGGagcTGATGTGTATTCTGT & \\
\hline & $\mathrm{F}$ & LG-15 & GTTGCAATCAAGCCC $g c t$ TGCACAGATGGTGTG & \\
\hline & $\mathrm{R}$ & LG-16 & CACACCATCTGTGCA $a g c$ GGGCTTGATTGCAAC & \\
\hline \multirow{4}{*}{$\begin{array}{c}\text { pFastbac1-GP64 } \\
\text {-6xHis-NSvc2 } \\
\text {-1xFlag }\end{array}$} & $\mathrm{F}$ & P3209 & $\begin{array}{c}\text { CCggatccATGCACCACCACCACCACCACCCAAT } \\
\text { ACCTTTCCCTGACAC }\end{array}$ & \multirow{4}{*}{$\begin{array}{c}\text { To amplify RSV } \\
\text { NSvc2 and GP64 } \\
\text { fragments, then } \\
\text { clone into } \\
\text { pFastbac1 }\end{array}$} \\
\hline & $\mathrm{R}$ & P4501 & $\begin{array}{c}\text { CGctcgagTTACTTATCATCATCATCCTTGTAATC } \underline{\text { AT }} \\
\text { CAACCTGTCTAATGTC }\end{array}$ & \\
\hline & $\mathrm{F}$ & P3253 & CGggatccATGGTAAGCGCTATTGTTTT & \\
\hline & $\mathrm{R}$ & P3254 & GAagatetGTTGCAGTGCTCCGCCGCAA & \\
\hline \multirow{8}{*}{$\begin{array}{c}\text { pFastbac1-GP64 } \\
\text {-6xHis-NSvc2(N114A/ } \\
\text { N199A/N232A) } \\
\text {-1xFlag }\end{array}$} & $\mathrm{F}$ & $\mathrm{P} 4726$ & CCACCATCGGGCGCGGATCCatggtaagcgctattgtttt & \multirow{8}{*}{$\begin{array}{l}\text { To amplify point } \\
\text { mutant fragment } \\
\text { and vector } \\
\text { fragment, then do } \\
\text { homologous } \\
\text { recombination to } \\
\text { obtain plasmid }\end{array}$} \\
\hline & $\mathrm{R}$ & P4727 & aaaacaatagcgcttaccatGGATCCGCGCCCGATGGTGG & \\
\hline & $\mathrm{F}$ & LG-5 & GATGCTATTCTGCAGgctGTATCTCTGGTTGGC & \\
\hline & $\mathrm{R}$ & LG-6 & GCCAACCAGAGATACagcCTGCAGAATAGCATC & \\
\hline & $\mathrm{F}$ & LG-7 & TCTGCATGTGAGGTCgctGTGAGTGACCAGACA & \\
\hline & $\mathrm{R}$ & LG-8 & TGTCTGGTCACTCACagcGACCTCACATGCAGA & \\
\hline & $\mathrm{F}$ & LG-9 & AAGGGTGGTTGTCAA $g c t$ GTTACTTGTCACCCA & \\
\hline & $\mathrm{R}$ & LG-10 & TGGGTGACAAGTAACagcTTGACAACCACCCTT & \\
\hline
\end{tabular}




\begin{tabular}{|c|c|c|c|c|}
\hline \multirow{8}{*}{$\begin{array}{c}\text { pFastbac1-GP64 } \\
\text {-6xHis-NSvc2(F460A/ } \\
\text { F489A/Y498A) } \\
\text {-1xFlag }\end{array}$} & $\mathrm{F}$ & $\mathrm{P} 4726$ & CCACCATCGGGCGCGGATCCatggtaagcgctattgtttt & \multirow{8}{*}{$\begin{array}{l}\text { To amplify point } \\
\text { mutant fragment } \\
\text { and vector } \\
\text { fragment, then do } \\
\text { homologous } \\
\text { recombination to } \\
\text { obtain plasmid }\end{array}$} \\
\hline & $\mathrm{R}$ & $\mathrm{P} 4727$ & aaaacaatagcgcttaccatGGATCCGCGCCCGATGGTGG & \\
\hline & $\mathrm{F}$ & P2603 & GAGGAATGGAAATGTgctGAAGGGGAAGGTTGC & \\
\hline & $\mathrm{R}$ & $\mathrm{P} 2604$ & GCAАССТTCСССТTCagcACATTTCCАТTCСТC & \\
\hline & $\mathrm{F}$ & P2605 & TATTGTGTCACTGAT $g c c$ CACATATTTTCCTAT & \\
\hline & $\mathrm{R}$ & $\mathrm{P} 2606$ & ATAGGAAAATATGTG $g g c$ ATCAGTGACACAATA & \\
\hline & $\mathrm{F}$ & P2613 & TCCTATTGCCCAGCA $g c c$ CACTACAATTGGAAG & \\
\hline & $\mathrm{R}$ & P2614 & CTTCCAATTGTAGTGggcTGCTGGGCAATAGGA & \\
\hline
\end{tabular}

981 The terminal fusion sequences in the homologous recombination primers are in 982 uppercase and lowercase letters; the restriction enzyme sites are the lowercase letters;

983 the 1xFlag sequence is the italic letters; the gene sequences are underlined, and the 984 point mutation sites are the lowercase italic letters. 